University of Tennessee Health Science Center

UTHSC Digital Commons

\title{
Mapping the Spatial and Temporal Dynamics of Sensorimotor Integration During the Perception and Performance of Wallowing
}

\author{
Megan E. Cuellar \\ University of Tennessee Health Science Center
}

Follow this and additional works at: https://dc.uthsc.edu/dissertations

Part of the Medical Physiology Commons, Other Analytical, Diagnostic and Therapeutic Techniques and Equipment Commons, and the Speech and Hearing Science Commons

\section{Recommended Citation}

Cuellar, Megan E. , "Mapping the Spatial and Temporal Dynamics of Sensorimotor Integration During the Perception and Performance of Wallowing" (2014). Theses and Dissertations (ETD). Paper 50. http://dx.doi.org/10.21007/etd.cghs.2014.0060. 


\title{
Mapping the Spatial and Temporal Dynamics of Sensorimotor Integration During the Perception and Performance of Wallowing
}

\author{
Abstract \\ Similar to other complex sequences of muscle activity, swallowing relies heavily upon 'sensorimotor \\ integration.' It is well known that the premotor cortex and primary sensorimotor cortices provide critical \\ sensorimotor contributions that help control the strength and timing of swallowing muscle effectors. \\ However, the temporal dynamics of sensorimotor integration remains unclear, even when performed \\ normally without neurological compromise. Recent advances in EEG analysis blind source separation \\ techniques via independent component analysis offer a novel and exciting opportunity to measure \\ cortical sensorimotor activity in realtime during swallowing, concurrently with muscle activity during \\ swallow initiation. In the current study, mu components were identified, with characteristic alpha $(\sim 10 \mathrm{~Hz})$ \\ and beta $(\sim 20 \mathrm{~Hz})$ frequency bands. Spectral power within these frequency bands are known to index \\ somatosensory and motor activity, respectively. Twenty-five adult participants produced swallowing and \\ tongue tapping (motor control) tasks. Additionally they were asked to watch a video depicting swallowing \\ and a scrambled kaleidoscope (perceptual control) version of this same video. Independent component \\ analysis of raw EEG signals identified bilateral clusters of mu components, maximally localized to the \\ premotor cortex (BA6) in 19 participants during the production and the perception tasks. Event related \\ spectral perturbation (ERSP) analysis was used to identify spectral power within alpha and beta peaks of \\ the mu cluster across time. Alpha and beta event-related desynchronization (ERD), indicative of \\ somatosensory and motor activity, was revealed for both tongue tapping and swallowing beginning at \\ $\sim 500 \mathrm{~ms}$ following a visual cue to "go." However, the patterns of ERD are stronger (pFDR

\section{Document Type \\ Dissertation} \\ Degree Name \\ Doctor of Philosophy (PhD) \\ Program \\ Speech and Hearing Science \\ Research Advisor \\ Tim Saltuklaroglu, Ph.D. \\ Keywords \\ electroencephalography, event related desynchronization, mu rhythm, swallowing

\section{Subject Categories} \\ Medical Physiology | Medicine and Health Sciences | Other Analytical, Diagnostic and Therapeutic \\ Techniques and Equipment | Speech and Hearing Science
}


Mapping the Spatial and Temporal Dynamics of Sensorimotor Integration during the Perception and Performance of Swallowing

\author{
A Dissertation \\ Presented for \\ The Graduate Studies Council \\ The University of Tennessee \\ Health Science Center
}

\author{
In Partial Fulfillment \\ Of the Requirements for the Degree \\ Doctor of Philosophy \\ From The University of Tennessee
}

By

Megan E. Cuellar

December 2014 
Copyright (C) 2014 by Megan Elizabeth Cuellar.

All rights reserved. 


\section{DEDICATION}

I would like to dedicate this dissertation to my family and friends. To my mother, Sally Davenport, your love, support, and quiet strength have carried me through the last six years. Mom, you are the "wind beneath my wings." I can never express my gratitude for all that you have given to help me become the woman that I am today. To my father, Dale Morris, you have always been my hero. Your love, wisdom, strength, and support have provided me with the foundation of my life as well as a sense of direction throughout this journey. To my husband, Jeff Cuellar, your love, kindness, strength, laughter, joy, encouragement, and companionship allowed me to achieve this accomplishment. I love you more than words can express; you are my everything. To my son, Kingston Cuellar, you are such an amazing blessing. I am in awe of your beautiful spirit. I love all that you are and everything that you will become. Thank you for giving immeasurable joy and inspiration along the way. Lastly, to my friends and especially to Jennine Harvey-Northrop, your sense of humor, fierce support, creativity, intelligence and grit inspired me to achieve this goal. Thank you all for sharing this adventure with me. 


\section{ACKNOWLEDGEMENTS}

I would like to thank the chair of my committee, Dr. Tim Saltuklaroglu whose endless patience, poignant insight, and expertise made it possible for me to achieve this goal. I would also like to thank my committee members, Dr. Ashley Harkrider, Dr. Kristin King, and Dr. John Boughter, for agreeing to serve on my committee and for offering your support, guidance, and feedback along the way. I would like to acknowledge Dave Jenson and Dave Thornton whose assistance with running study participants and everyday humor made life in the lab a true joy. Finally, I would like to acknowledge the University of Tennessee Health Science Center for allowing me to pursue my goal of obtaining a Ph.D. in speech pathology. 


\begin{abstract}
Similar to other complex sequences of muscle activity, swallowing relies heavily upon 'sensorimotor integration.' It is well known that the premotor cortex and primary sensorimotor cortices provide critical sensorimotor contributions that help control the strength and timing of swallowing muscle effectors. However, the temporal dynamics of sensorimotor integration remains unclear, even when performed normally without neurological compromise. Recent advances in EEG analysis blind source separation techniques via independent component analysis offer a novel and exciting opportunity to measure cortical sensorimotor activity in real-time during swallowing, concurrently with muscle activity during swallow initiation. In the current study, mu components were identified, with characteristic alpha $(\sim 10 \mathrm{~Hz})$ and beta $(\sim 20 \mathrm{~Hz})$ frequency bands. Spectral power within these frequency bands are known to index somatosensory and motor activity, respectively. Twenty-five adult participants produced swallowing and tongue tapping (motor control) tasks. Additionally they were asked to watch a video depicting swallowing and a scrambled kaleidoscope (perceptual control) version of this same video. Independent component analysis of raw EEG signals identified bilateral clusters of mu components, maximally localized to the premotor cortex (BA6) in 19 participants during the production and the perception tasks. Event related spectral perturbation (ERSP) analysis was used to identify spectral power within alpha and beta peaks of the mu cluster across time. Alpha and beta event-related desynchronization (ERD), indicative of somatosensory and motor activity, was revealed for both tongue tapping and swallowing beginning at 500 ms following a visual cue to "go." However, the patterns of ERD are stronger $(p \mathrm{FDR}<.05)$ in the right mu cluster in swallowing vs. tongue tapping. These findings were interpreted as being indicative of normal sensorimotor loops involved in swallowing arising from the need to produce complex motor movements with accurate guidance and feedback. Beta ERD is speculated to index motor activity both from muscle contraction and internal modeling, while alpha ERD is thought to index feedback to premotor cortex, helping to update internal models and guide future movements. The findings in this study are consistent with notions that the premotor cortex functions as a sensorimotor processing hub for feedforward and feedback driven sensorimotor loops necessary in motor control. Alpha and beta ERD are revealed when participants watched videos of swallowing, and was stronger $(p \mathrm{FDR}<.05)$ than when watching the control video. It is posited that watching biologically relevant videos (e.g., swallowing) induces a form of covert replay, which induces similar patterns of sensorimotor activity when compared to patterns of sensorimotor activity during the performance of swallowing. Overall, the results of this study suggest that using the novel techniques performed in this study offer exciting opportunities to measure functional changes in mu ERD during swallowing, which may be applied to provide better understanding of the effects that various phase-specific (oral vs. pharyngeal and esophageal) swallowing impairments have upon the integrity of feedforward and feedback driven SMI during swallowing. In doing so, perhaps a better understanding may be obtained regarding the therapeutic effects of sensorimotor swallowing treatments upon cortical SMI during swallowing.
\end{abstract}




\section{TABLE OF CONTENTS}

CHAPTER 1. INTRODUCTION ................................................................................

CHAPTER 2. LITERATURE REVIEW ........................................................................7

Anatomy and Physiology of the Peripheral Swallowing Mechanism ...........................

The Neurophysiology of Swallowing .................................................................

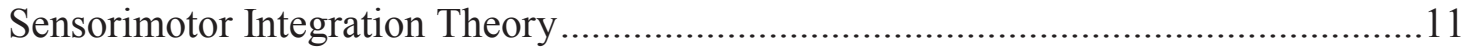

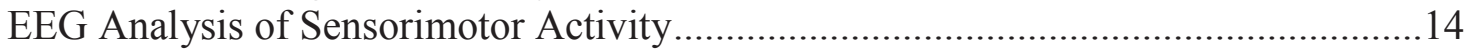

EEG: Independent Component Analysis ............................................................... 15

Using EEG to Analyze Changes in Regional Neural Activity Across Time.................17

$\mathrm{Mu}$ Cluster Activity During Different Phases of Swallowing ...................................18

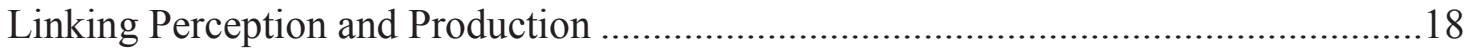

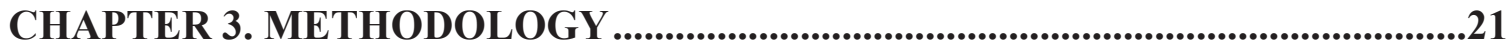

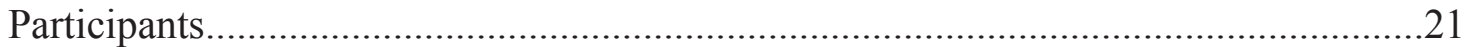

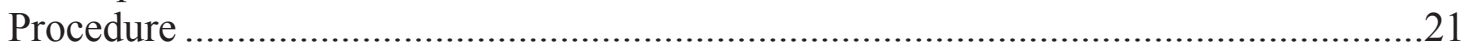

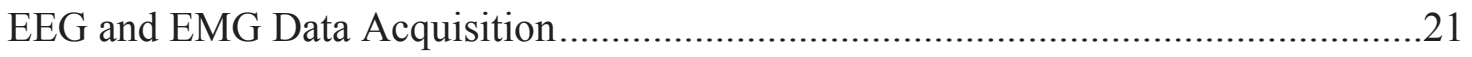

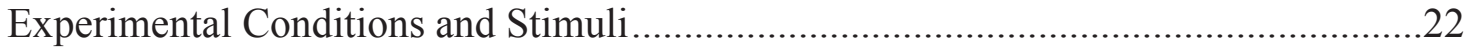

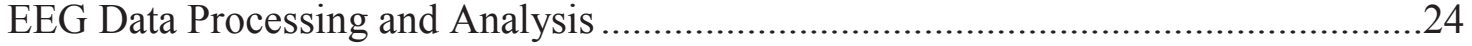

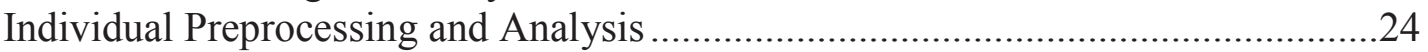

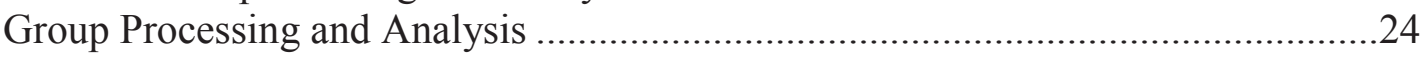

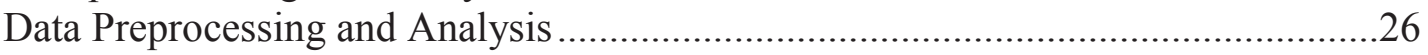

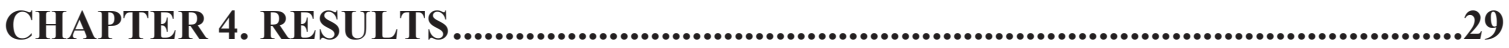

Performance Conditions: Independent Component Clustering .................................29

Performance Conditions: Event Related Spectral Perturbations (ERSP) ....................29

Perception Conditions: Independent Component Clustering......................................34

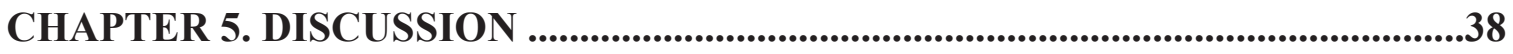

Aim 1: Using ICA to Identify Sensorimotor and Muscle Activity ..............................39

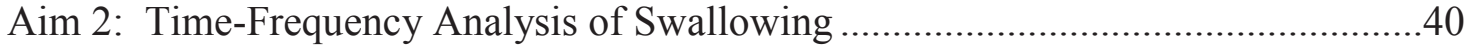

Mu-alpha and Mu-beta Across the Time Course of the Swallow ..............................40

Mu-alpha and Beta During the Oral vs. Pharyngeal and Esophageal Phases ............41

Sensorimotor Integration in Swallowing vs. Tongue Tapping ...............................42

Aim 3: Descriptive Comparison of Swallowing Perception and Performance ............43

Mu-alpha and Mu-beta During Perception: Swallowing vs. Kaleidoscope..............44

Mu-alpha and Beta: Swallowing Perception vs. Swallowing Performance..............45

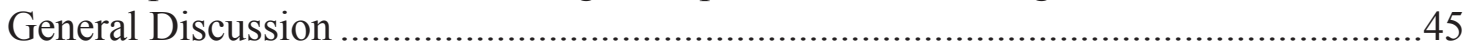

Limitations and Future Directions of Study .................................................. 47

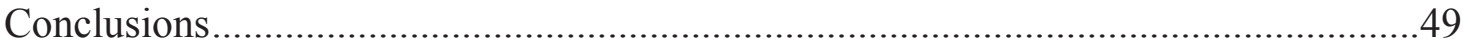

LIST OF REFERENCES .....................................................................................................50

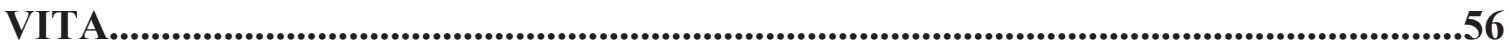




\section{LIST OF FIGURES}

Figure 1. Still Frame Pictures Taken from the Kaleidoscope Video..........................23

Figure 2. Still Frame Pictures Taken from the Animated Swallowing Video .............23

Figure 3. Timeline of Kaleidoscope and Swallowing Perception Condition ..............25

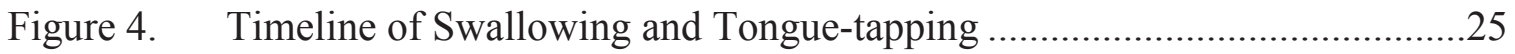

Figure 5. Epoched Timeline for All Four Perception and Performance Conditions ....25

Figure 6. Right Mu Spectral Displays for Swallowing and Tongue-tapping ...............30

Figure 7. Left Mu Spectral Displays for Swallowing and Tongue-tapping ................31

Figure 8. Right and Left Mu IC Cluster Average ECD's and ERSP alongside EMG Cluster ECD and ERSP ............................................................ 32

Figure 9. Right Mu Spectral Displays for Swallowing and Kaleidoscope

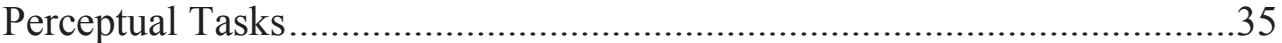

Figure 10. Left Mu Spectral Displays for Swallowing and Kaleidoscope Perceptual

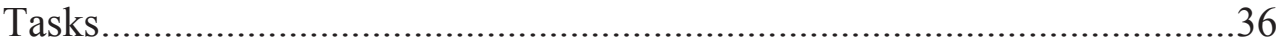

Figure 11. Left Mu IC Cluster Average ECD for Kaleidoscope and Swallowing Perceptual Tasks ........................................................................... 37

Figure 12. Right $\mathrm{Mu}$ IC Cluster Average ECD for Kaleidoscope and Swallowing Perceptual Tasks..... 


\section{LIST OF ABBREVIATIONS}

\begin{tabular}{ll} 
BA & Brodmann's area \\
BEM & Boundary element Model \\
BOLD & Blood oxygen level dependent \\
BSS & Blind source separation \\
Cb & Cerebellum \\
CN's & Cranial Nerves \\
CPG & Central patterns generator \\
ECD & Equivalent current dipole \\
ECG & Electrocardiographic \\
EEG & electroenceophalography \\
EMG & Electromyography \\
EOG & Electrooculogram \\
ERD & Event related desynchronization \\
ERS & Event related synchronization \\
ERSP & Event related spectral perturbations \\
FDR & False Discovery Rate \\
FEES & Fiberoptic endoscopic evaluation of swallowing \\
fMRI & Functional magnetic resonance imaging \\
HEOG & Electrooculogram electrode (right) \\
Hz & Hertz \\
ICA & Independent Component Analysis \\
IC's & Independent Components \\
IRB & Institutional Review Board \\
ITI & Intertrial Interval \\
k $\Omega$ & Kilohms \\
M1 & Mastoid 1 \\
M2 & Mastoid 2 \\
MCA & Middle Cerebral Artery \\
MEG & Magnetoencephalography \\
MN & Mirror neurons \\
ms & miliseconds \\
NA & Nucleus ambiguous \\
NBS & Neurobehavioral systems \\
NTS & Nucleus tractus solitaries \\
PCA & Principle component analysis \\
PD & Parkinson's Disease \\
PET & positron emission tomography \\
PMC & Premotor cortex \\
RV & Residual variance \\
sEMG & Surface electromyography \\
SMI & Sensorimotor Integration \\
SLORETA & Standardized low resolution tomography \\
TMS & Transcranial stimulation \\
& \\
\hline
\end{tabular}


VEOG

Electrooculogram electrode (left)

VFSS

Videoflouroscopy 


\section{CHAPTER 1. INTRODUCTION}

The ability to swallow is vital to feeding and therefore necessary for survival. Swallowing is achieved by precisely coordinating the contraction and relaxation of more than 50 muscles during the oral, pharyngeal, and esophageal phases of swallowing (Ertekin \& Aydogdu, 2003; Shaw \& Martino, 2013; Hamdy, 2013). In order to successfully perform the necessary action sequences in an efficient manner, high level neuromuscular control is required, continuously integrating sensory (e.g., thermal, gustatory, proprioceptive, and somatosensory) information with the execution of muscle movements (Ertekin \& Aydogdu, 2003; Hamdy et al., 1999; Langmore, 2001; Mosier \& Bereznaya, 2001; Furlong et al., 2004; Logemann, 1998; Shaw \& Martino).

Swallowing begins with the 'oral preparatory' and the 'oral transit' phases which collectively form the 'oral' phase (Logemann, 1998). In the oral phase, food and/or liquid is transformed into a bolus that is subsequently propelled posteriorly toward the pharynx (Logemann, 1998). As the bolus reaches the anterior faucial pillars, the pharyngeal phase of swallowing 'triggers' to propel the bolus through the pharynx via pressure forces generated from hyolaryngeal excursion (Logemann, 1998; Langmore, 2001). The pharyngeal phase of swallowing is easily observed using imaging techniques such as videoflouroscopy (VFSS), fiberoptic endoscopic evaluation of swallowing (FEES), or surface electromyography (sEMG) (Logemann, 1997; Langmore, 2001; Perlman, Schulze, \& Delerie, 1998). However, the neurological control of these events is less well understood, especially in regards to the control over timing and coordination of muscular activity. This gap in knowledge is critical considering that most swallowing disorders result from neurological damage as opposed to compromises in the peripheral swallowing mechanism (NINDS, 2013).

Imaging studies have shown bilateral activity across numerous cortical and subcortical structures during swallowing. These include the premotor cortex (PMC) and supplementary motor area (Brodmann's area (BA 6), the primary motor cortex, the primary somatosensory cortex (BA 1, 2, 3), the superior temporal sulcus (BA 22 ), the parietal sensory association areas (BA 5 and 7), the insula (BA 14, 16 ), the cingulate cortex (BA 24), the basal ganglia, the cerebellum, and the brain stem (Ertekin \& Aydogdu, 2003; Hamdy et al., 1999; Martin, Goodyear, Gati, Mennon, 2000; Zald \& Pardo, 1999). The functional contributions of these regions to swallowing are less wellunderstood, especially with regards to the timing of activity across the oral and pharyngeal phases of swallowing. Cortical contributions to the oral vs. pharyngeal phase of swallowing are thought to differ because the oral phase is under voluntary control while the pharyngeal phase is partially reflexive (Jean, 1984; Jean 2001). Furthermore, shared patterns of cortical activity are often observed during the performance of similar oral-motor tasks that mimic some of the movements performed during swallowing such as tongue-tapping, tongue tip elevation, lip puckering, base of tongue retraction, and vocal fold adduction that mimic (Humbert \& Robbins, 2007; Malandraki, Sutton, Perlman, \& Karampinos, 2010; Martin et al., 2004). Therefore, including these tasks in 
swallowing studies helps dissociate the cortical activity that occurs in swallowing from the cortical activity that is generated when performing other related oral-motor tasks.

Similar to other complex sequences of muscle activity, swallowing relies heavily upon 'sensorimotor integration' (SMI), (Humbert, Lockerston, Christopherson, German, \& Stone, 2012; Humbert \& German, 2013; Mosier et al., 1999; Rao et al., 1997; Zald \& Pardo, 1999). SMI is a higher order neurophysiological process that interprets, associates, and modulates multimodal sensory information in order to provide adaptive, on-line adjustments to movements (Ayers, 1972; 1989). When swallowing is initiated, motor commands are sent to muscle effectors to produce movements. When structures begin moving, neural impulses conveying the sensory consequences of the movements are sent back to motor regions and interpreted to shape subsequent movements. However, it is well-documented that if motor control was to rely solely on the afferent feedback from muscle effectors, the entire system would function too slowly to generate online motor corrections when necessary (Wolpert, Diedrichsen, \& Flanagan, 2011). Online motor corrections to adjust the strength and timing of muscle activity may be performed during swallowing for a variety of reasons. For example, an unexpectedly large volume of food or liquid may be accepted, which requires additional strength of muscle activity to form and transition the bolus through the oral cavity and pharynx. Additionally, if the large volume of food or liquid entered into the oral cavity quicker than expected, then the pharyngeal swallow phase needs to 'trigger' earlier than originally anticipated. Thus, in addition to this 'externally driven' feedback loop, an 'internally driven' feedback loop provides more immediate feedback to help make online corrections in motor control based upon predictions about the sensory outcome of movement (Kawato, 1999; Wolpert \& Flanagan, 2001). That is, as a motor command is sent to the musculature, an internal model, i.e. efference copy of the motor plan coupled with the 'predicted' sensory consequences of movement, is sent directly from premotor regions to somatosensory regions. (Wolpert, Ghahramani, \& Flanagan, 2001). The sensory predictions are compared with the goal of the movement that is coded in the somatosensory regions so corrective adjustments can be relayed back to the PMC before the actual sensory consequences of a movement are registered (Kalaska, Scott, Cisek, \& Sergio, 1997; Ito, 2008; Schubotz et al., 2008). As information from the actual sensory consequences is received from the musculature, this information is incorporated into the reafferant feedback to the PMC. Hence the outflow of motor information, i.e. motor commands to peripheral swallowing muscles and the efference copy of swallowing, and the inflow of reafferent sensory feedback allow the PMC and the adjacent primary motor and somatosensory regions to form an important hub for sensorimotor processing during swallowing.

Cortical sensorimotor activity during swallowing has been previously measured using fMRI or PET, which offer excellent spatial but poor temporal resolution. EEG offers excellent temporal resolution; however, employing EEG techniques to measure sensorimotor activity in swallowing has been significantly limited due to poor spatial resolution stemming from volume conduction and contamination of neural signals from muscle artifact in motor tasks. However, recent advancements in EEG analysis techniques offer a new time-sensitive means of measuring sensorimotor control in 
swallowing. The 'mu rhythm' has been found to emanate predominantly from sensorimotor regions and is characterized by spectral peaks in the $\sim 10 \mathrm{~Hz}$ 'alpha' and $\sim 20$ Hz 'beta' frequency bands, (Chen, Bin, Daly, Gao, 2013; Hari, 2006; Jensen, Goel, Kopell, Pohja, Hari \& Ermentrout, 2005; McGarry, Russo, Schalles \& Pineda, 2012; Pineda, 2005; Pineda, 2008;). Decreases in spectral power (i.e., desynchronization) of the EEG mu rhythm has long been associated with sensorimotor activity. Specifically, decreases in beta power are considered indications of motor activity and decreases in alpha power are considered to be indications of somatosensory activity. These notions are somewhat consistent with traditional understanding of alpha and beta EEG oscillations. The alpha frequency band is generally considered a measure of sensory perception, and has been found to suppress in response to auditory, visual, and tactile stimulation (Palva \& Palva, 2010). Additionally, several studies have reported mu-alpha band suppression in response to applied proprioceptive stimulation to the hands (Cheyne et al., 2003), as well as the thumbs, toes, and lips (Gaetz \& Cheyne, 2005). Additionally, the beta frequency band provides a general index of motor activity (Kilavik, Zaepfill, Brovelli, \& MacKay, 2013; Neuper, Wortz, Pfurtscheller, 2006; Tzagarakis, Ince, Leuthold, Pellizzer, 2010). In studies of cued movement performance, mu-beta band suppression has been found prior to the onset of finger tapping (Pfurtscheller, Zollaudek, $\&$ Neuper, 1996) and grasping movements (McFarland et al., 2000), and in accordance with feed-forward internal modeling theory is interpreted as evidence of predictive motor activity associated with activating the efference motor copy of movements (Obai, Mari, Vorbach, Hallett, 2005; Neuper et al., 2006; Siegel et al., 2012). Mu rhythm beta suppression has also occured during the performance of isotonic as well as dynamic selfpaced movements, and is thought to regulate the timing and intensity (i.e. strength) of muscle activity during movement execution (Crone et al., 1998; Doyle, Yarrow, Brown, 2005). Considering that examining the spectral power of the mu rhythm within its characteristic peaks has been taken as a measure of sensorimotor integration in during other dynamic movement tasks, it therefore seems reasonable to use similar methods to measure SMI during swallowing.

Blind source separation (BSS) of raw EEG signals using independent component analysis (ICA) provides a means to localize and separate cortical sensorimotor activity from muscular activity (McMennamin et al, 2011; Onton, Westerfield, Townsend, \& Makeig, 2012; Schelenz et al., 2013; Wagner et al., 2012). Based upon the assumption that the neural sources of EEG activity stream from temporally independent and spatially fixed sources, ICA separates mixtures of surface EEG activity into temporally independent and spatially fixed functional components (Delorme \& Makeig, 2004; Delorme, Senjowski, Makeig, 2007). Using ICA to estimate cortical sources of sensorimotor activity, a number of studies involving observation, imagination, and performance of movement, have identified mu components that are localized to the PMC and primary sensorimotor cortices (Arnstein, Cui, Keysers, Mauritz, Gazzola, 2011; Kourtis, De Saedeleer, Vingerhoets, 2014; McFarland et al., 2000; Paek, Harshavardhan, Agashe, Contreras-Vidal, 2014; Wagner et al., 2012). Additional studies involving the perception of tactile and somatosensory information have reported mu components localized to the sensorimotor and sensory association areas (Gaetz \& Cheyne, 2006; Palva et al., 2005; Quandt, Marshall, Bouquet, Shipley, 2013). Based on recent findings 
from studies of SMI during speech perception and production processes (e.g., Bowers et al., 2013; Jenson et al., 2014), which are known to also elicit cortical sensorimotor activity in the PMC and primary sensorimotor cortices, it therefore seems reasonable to predict that ICA techniques will provide an effective means to identify and localize SMI during the performance of swallowing. Once mu components are identified via ICA, time-frequency analyses can provide measures of sensorimotor activity across time. Event-related spectral perturbation (ERSP) analysis offers an ideal means to analyze quantitatively patterns of event-related synchronization (ERS), and event-related desynchronization (ERD), providing time-sensitive measures of spectral power in EEG components (Onton et al., 2007; Pfurtscheller \& Silva, 1999) that reflect activity within neural components across time. For example, Bowers et al. (2013) analyzed changes in the spectral power of mu components during auditory discrimination tasks to better understand the functional role of SMI during speech perception. Additional studies involving the kinesthetic perception of driving simulations (Chuang Ko, Jung, \& Lin, 2014), audiovisual perception of emotional faces (Schelenz et al., 2013), working memory recall of shapes and letters (Lenartowicz et al., 2014), and word reading tasks (Bedo, Ribary, \& Ward, 2014) have used ERSP to measure dynamic changes in the power of neural components across time. Cumulatively, these studies suggest that using ERSP to map the changes in the strength of sensorimotor activity in real time during swallowing.

Until recently, very few EEG studies have included tasks that require 'active' or voluntary muscular contraction, because muscle activity related to eye blinking and other head and neck muscle movements (i.e. muscle artifacts) are known to reduce the signalto- noise ratio and overwhelm the spectral characteristics of neural signals (McMenamin et al., 2011). Furthermore, due to volume conduction, muscle artifacts can contaminate data recorded from every electrode, possibly decreasing localization accuracy (Jung et al., 2000; Mima \& Hallett; 1999; van den Broek, Reinders, Donderwinkel, \& Peters; 1998). ICA addresses these concerns since muscle and neural activity emanate from distinct sources and therefore are separable using ICA. However, when using ICA to collect neural and muscle activity during the performance of movement tasks, it has been noted that muscle components represent a majority of the total signal variance, resulting in a reduction of the spectral power exhibited by the neural components (McMenamin et al., 2011; Vos et al., 2010). Despite these possible limitations, a number of studies have reported successful use of an ICA for identifying neural activity during arm movements (Alomari, Samaha, \& AlKamha, 2013; Hausser, 2006), finger tapping and hand movements (Lv, Li, Gu, 2010), walking and running movements (Gwin et al., 2010; Lau et al., 2014), and postural adjustments (Slobounov, Cao, Jaiswal, \& Newell, 2010), providing evidence that ICA can indeed effectively separate neural from non-neural signals and supporting its use in this study. Taking it a step further, recently Jenson and colleagues used ICA to measure concurrently "in head" mu rhythm activity and "out of head" EMG muscle activity associated with lip movements, which provided a physiological reference to mark the onset and offset of bilabial syllable and multisyllabic speech productions (Jenson et al., 2014). This technique has not been used previously during swallowing, however, considering that speech and swallowing both involve 
temporal sequences of complex movements in the oral cavity and vocal tract, it seems logical to use the same technique in this study.

Surface electromyography (sEMG) is used frequently to identify muscle activity associated with the pharyngeal phase of swallowing. For example, sEMG measures of submental activity are used to identify physiological events occurring during the oral and pharyngeal phases of swallowing while sEMG measures of infrahyoid activity reflect muscle activity during the pharyngeal and esophageal phases of swallowing (Ding, Larson, Logemann, \& Rademaker, 2001; Perlman, Palmer, McCulloch, \& Vandaele, 1998; Viaman, Eviatar, Segal, 2004, Viaman et al., 2009). Measuring infrahyoid muscle activity during swallowing provides a physiological reference that temporally marks pharyngeal phase muscle activity. Although infrahyoid activity doesn't provide information regarding the transition between the oral and pharyngeal phases, it does allow for interpretations specific to pharyngeal phase without influences from the oral phase. Considering the prevalence of myogenic components found in ICA, it seems likely that placing EMG electrodes on the infrahyoid muscle complex will identify muscle activity via ERS spreading across all measured frequencies during the pharyngeal and esophageal phases of swallowing. Activity in this component is expected to be reduced or absent in the non-swallowing control task. Most importantly this muscular activity can be used as a temporal reference when interpreting ERS (muscle activity) and ERD (neural activity) in the mu rhythm. Thus, ERSP analysis of this component, alongside the mu components, will temporally demarcate neural activity associated with sensorimotor control in the oral and pharyngeal phases of swallowing.

Beyond mapping the time-course SMI during the performance of swallowing, the relationship between the perception and performance of swallowing behavior is also of interest. A large body of evidence suggests a shared sensorimotor representation of the perception (action observation) and production (action execution) of biologically relevant motor actions (Rizzolatti, Fadiga, Gallese, \& Fogassi, 1996; Rizzolatti \& Craighero, 2004, Rizzolatti \& Fabbri-Destro 2008). Some suggest that the functional link between the auditory and/or visual perception of motor actions and the performance of motor actions provides the foundation for imitation-based motor learning (Fadiga, Craighero, \& Oliverier, 2005; Le Bel, Pineda, \& Sharma, 2009; Pineda, 2005). Specifically, the ability to observe, interpret, and integrate the auditory and/or visual perception of an action with the efference motor copy representation of the same action is thought to strengthen and/or modify an individual's internal (model) representation of an action (Pineda, 2005; Rizzioloatti \& Craighero, 2004; Zaepffel, Trachel, Kilvik, \& Brochier, 2013). Thus, identifying a shared representation of cortical sensorimotor activity, measured via changes in the Mu rhythm, during the perception and performance of swallowing may provide insight regarding the potential of observation or imitation based treatment approaches to facilitate the acquisition, mastery, and restoration of swallowing behavior.

Temporal information is needed to better understand the functional role of SMI during the performance and perception of swallowing. The mu rhythm represents a rich source of SMI information, because it provides a global measure of sensory and motor processing that can be measured in different cortical regions known to mediate 
swallowing. Moreover, ERSP analyses can provide measures of sensorimotor activity and infrahyoid muscle activity can be analyzed across time to allow for interpretations of SMI that are specific to particular phases of interest.

Despite significant improvements in neuroimaging techniques, the functional and temporal parameters of sensorimotor contributions to normal human swallowing behavior remain unclear. Identifying and interpreting the time course of the EEG mu rhythm ERS /ERD offers a novel means for examining the cortical sensorimotor control of swallowing. The overarching goals of the study are twofold. First, they are to use ICA to identify sensorimotor mu components in the perception and production of swallowing. Upon accomplishing this, the second goal will be to use ERSP to provide measures of sensorimotor activity across time that can be examined concurrently with infrahyoid muscle activity. In doing so, the results may help to specify phase-specific functional contributions of SMI during oropharyngeal swallowing. In addition, these data will provide information regarding the functional link between the performance and the perception of normal swallowing behavior. 


\section{CHAPTER 2. LITERATURE REVIEW}

\section{Anatomy and Physiology of the Peripheral Swallowing Mechanism}

Swallowing is a vital behavior that when impaired can negatively impact a variety of health-related factors such as nutritional status, medical stability, level of independence, and/or quality of life for an individual (Barer, 1989; Kayser-Jones \& Pengilly, 1999; Lam \& Lai, 2011;). The ability to swallow depends upon the timely detection, interpretation, and response to a multitude of sensory signals derived from thermal, gustatory, tactile, and proprioceptive sensory information (Jafari, Prince, Kim, Paydarfar, 2004; Steele \& Miller, 2010; Steele, 2013). Motoricly, swallowing can be described as a complex series of sequentially and temporally organized movements that require both voluntary and involuntary control (Ertekin \& Aydogdu, 2003; McNeil, 2009; Miller 2001). Thus, the ability to swallow safely and efficiently depends upon the timely detection, interpretation, and modulation of afferent input, i.e. reaffarent sensory information, as well as efferent motor output, sent to muscles of the oropharynx (Jafari et al., 2004).

Transitioning a bolus, or a well-formed cohesive ball of food or liquid, from the oral cavity to the esophagus, requires 55 oral, pharyngeal, laryngeal, and esophageal muscles along with 6 cranial nerves (Ertekin \& Aydogdu 2003; Hani-Shaker, 2013; Lim, Lee, Lim, \& Choi, 2009). Physiologically, swallowing is divided into three functional phases, the oral phase (i.e. oral preparatory and oral phase), the pharyngeal phase, and the esophageal phase. Individual phases of swallowing are differentiated from one another according to the location of the anatomical structures involved in movement, as well as the time-course of physiological events (Logemann, 1989). As such, swallowing behavior is often described, assessed, and studied in terms of the particular phase or phase(s) of interest.

Anatomically, the oral phase of swallowing involves the lips, teeth, hard and soft palates, mandible, floor of the mouth, tongue, anterior faucial pillars, and posterior faucial pillars (Langmore, 2001). Physiologically, oral structures work together to manipulate and move the bolus, or well-formed mass of food or liquid, from the oral cavity to the pharyngeal cavity (Langmore, 2006; Logemann, 1997). As the bolus reaches the anterior faucial pillars, a rich set of sensory receptors signal to initiate the pharyngeal swallow response (Logemann, 1989). Anatomically, the three constrictor muscles of the pharynx along with the lateral and posterior pharyngeal walls, the glossopharyngus muscle, and the tongue base frame the pharyngeal cavity (Langmore, 2001; 2006). Together, the muscles, cartilage, and ligaments of the pharynx, in conjunction with the hyoid bone, achieve the following temporally organized physiological patterns of pharyngeal swallow physiology: 1) velopharyngeal closure; 2) tongue base retraction; 3) anterior elevation of the hyoid bone and larynx; 4) laryngeal closure; 5) epiglottic deflection; 6) opening of the cricopharyngeal sphincter; and 7) progressive constriction of the pharyngeal sphincters (Langmore, 2001). Through the sequentially organized manipulation of pharyngeal structures and passageways (listed 
above), the bolus travels from the pharynx to the esophagus (Logemann, 1997).

Maintaining the temporal and sequential order of physiological events, as the swallow progresses from the oral to the pharyngeal phase, is critical for safely and efficiently executing the swallow while maintaining upper airway protection (Kendall et al., 2000).

Given the importance of using phase-specific anatomical and physiological measures to define and describe normal swallowing, it is not surprising that disordered patterns of swallowing are also temporally described (Kendall et al., 2000; Moflenter \& Steele, 2012). Recent studies suggest that temporal measurements of swallowing physiology provide the most efficacious means to evaluate function quantitatively (Kendall et al., 2000; Lan et al., 2012; Martin-Harris et al., 2008; Miyaji et al., 2012; Stelle, Sasso, Bressman, 2012). For example, the duration of bolus transit times, the duration of physiological events, and the onset times of a physiological event are considered clinically significant measures for identifying and describing oral versus pharyngeal phase swallowing disorders (Curtis, Cruess, Dachman, \& Maso, 1984; Kendall et al., 2000; Ohmae, Logemann, Kaiser, Hanson, \& Kahrilas, 1995; Molfenter \& Steele, 2012). As a result, the diagnosis and treatment of phase-specific swallowing disorders is considered the optimal approach to diagnosing and treating swallowing disorders (Clark, 2003; Kays \& Robbins, 2006; Rofes, Villardell, Clave, 2013; Sugiyama et al., 2013; Steele, 2013).

The oral and pharyngeal phases of swallowing are easily viewed using instrumental methods to evaluate function. Videofluoroscopy (VF), considered by many to be the 'gold standard' for instrumental swallowing evaluations (Logemann, 1997; Langmore, 2001; Singh \& Hamdy, 2005) involves mixing food and liquid with a radiopaque substance, such as barium, to yield a contrasted view of swallowing behavior in real time (Aviv et al., 1996; Kidd, Lawson, Nesbitt, MacMahone, 1993; Martin et al., 1994; Martin-Harris et al., 2008). Using VFSS, the physiological distinction between the end of the oral phase and the beginning of the pharyngeal phase has been identified as occurring when the head of the bolus traverses the ramus of the mandible (Im, Kim, Oommen, Kim, Hwan-Ko, 2012; Logemann, 1997; Shaker, 2013; Stephen, Taves, Smith, \& Martin, 2005). Alternatively to VFSS, fiberoptic endoscopic evaluation of swallowing (FEES) provides a means to view and evaluate swallow physiology; however, there are notable differences in the imaging methods and viewing angles provided by FEES (Langmore, 2001). While VFSS is a radiographic imaging technique that offers a lateral view of oropharyngeal swallow physiology, fiberoptic endoscopy is a flexible scope with a camera that is passed through the nasopharynx into the pharynx, and provides a superior or 'bird's eye' view of the laryngeal vestibule (Langmore, 2001). Due to the different viewing angle provided by the FEES technique, the transition between the end of the oral phase and the beginning of the pharyngeal phase is marked when the arytenoids adduct (Langmore, 2001). Regardless of the differences between techniques (VFSS vs. FEES), both methods of instrumental assessment provide a reliable physiological distinction between the oral and pharyngeal phase of swallowing (Aviv et al., 1997; Aviv, 2000; Kelly, Leslie, Beale, Payton, Drinnan, 2006; Rees, 2006; Warnecke et al., 2009 ). 
Peripherally, the structural and physiological differences between the oral and the pharyngeal phases of swallowing are easily separated using instrumental techniques such as VFSS or FEES, which is important for both clinical evaluations and academic studies of swallowing behavior. However, there is limited knowledge regarding the time-course of oral versus pharyngeal phase neurophysiology due to the temporal limitations of traditional neuroimaging techniques such as functional magnetic resonance imaging (fMRI), computerized tomography (CT), or positron emission tomography (PET). Understanding the temporal dynamics of normal swallowing neurophysiology would provide an experimental foundation for the development of phase-specific diagnostic criteria and also would provide a better understanding of how sensory and motor treatments may remediate oral vs. pharyngeal phase swallowing disorders.

\section{The Neurophysiology of Swallowing}

Traditionally, swallowing was considered a reflexive behavior, controlled exclusively by brain stem structures (Miller, 2002). Functionally, a group of sensory and motor brain stem nuclei, known as the 'central pattern generator' (CPG) of swallowing, are known to 'trigger' and execute the pre-patterned pharyngeal swallow (Ertekin \& Aydogdu, 2003; Miller, 1996). The CPG of pharyngeal swallowing categorically can be divided into three functional systems: 1) the afferent sensory input system; 2) the efferent motor output system; and 3) the inter-neuronal network (Ertekin, 2010; Ertekin, 2011). Briefly, the nucleus tractus solitarius (NTS), located in the medulla of the brainstem is considered the main afferent system that receives sensory information from CN's IX \& X that triggers the pharyngeal swallow response (Jean, 1984). In response, interneurons alert the nucleus ambiguous (NA), representing the main efferent system, to send pre-patterned motor commands that transition the bolus through the pharynx while protecting the upper airway (Ertekin, 2010). Evidence of the CPG control of pharyngeal swallowing has been provided by decerebrate animal studies, as well as human lesion studies (Gordon, Hewer, \& Wade, 1987; Horner, Buoyer, Albers, \& Helms, 1991; Martino et al., 2005; Jean, 2001). Additionally, significant sensory and motor deficits affecting the oral preparation of the bolus, oral and pharyngeal bolus transit, and airway protection have been documented in a number of medullary brain stem lesion studies (Gordon, Hewer, \& Wade, 1987; Horner, Buoyer, Albers, \& Helms, 1991; Martino et al., 2005; Jean, 2001).

Although brainstem nuclei and cranial nerves are critical to swallowing, it is also known that subcortical regions such as the basal ganglia, thalamus, and cerebellum are essential to the temporal regulation and coordination of movements (Ertekin \& Aygodgdu, 2003; Martin, 2002). Epidemiology studies of stroke patients indicate that as many as $75 \%$ of individuals with thalamic lesions experience significant sensory deficits affecting oral bolus formation, oral and pharyngeal bolus transit, and airway protection (Cola et al., 2010; Hamdy, 2006). This is not surprising considering the diffuse connectivity between the thalamus and the sensory and motor regions in the cortex via cortico-thalamic pathways (Nolte, 2001; Haber \& Calzavera, 2009). The basal ganglia represents another well-known sensorimotor feedback processor that plays a crucial role 
in regulating oral bolus transit, the trigger of the pharyngeal swallow, pharyngeal bolus transit, and airway protection (Logemann, 2003). The effects of basal ganglia damage upon swallowing function are perhaps most apparent in individuals with dysphagia due to Parkinson's Disease (PD). According to a recent review, up to $87 \%$ of individuals with PD experience a myriad of symptoms including: impaired bolus formation, delayed oral transit, delayed trigger of the pharyngeal swallow; pharyngeal residue, aspiration, and aspiration pneumonia (Hoover, Baijens, Voskuilen, Oosterloo, \& Kremer, 2014). More recently, the cerebellum has been proposed to coordinate the temporal organization of swallowing (Martin et al., 2001). Although less documented by lesion studies, transcranial stimulation (TMS) studies show that stimulating the cerebellum increases excitability in the somatotopic area of the primary motor cortex that represents the pharynx (Vasant, Mistry, Jayasekeran, Michou, \& Hamdy, 2013). In addition, a recent animal study using a track-tracing technique showed increased spiking activity in the mediate deep cerebellar nucleus during fluid licking behaviors in mice (Lu, Cao, Tokita, Heck, \& Boughter, 2013). Taken together, the studies reviewed above demonstrate the importance of subcortical and cerebellar contributions to swallowing and offer some explanation for the highly variable profile of etiologies that may lead to dysphagia.

More recently, researchers have focused on identifying the cortical activity in swallowing. Using imaging techniques such as fMRI, PET, TMS, and MEG, clear evidence shows that swallowing is represented by an extensive distributed network of cortical sensory and motor regions (Erteken \& Aydogdu, 2003; Hamdy, 1999; Martin 2002; Miller, 1999; Soros, Inamoto, \& Martin, 2009). However, the functional dynamics of the cortical contributions to swallowing remain unclear. Even the question of hemispheric lateralization remains debated with some studies reporting bilateral patterns of hemispheric activity in the primary motor cortex, primary somatosensory cortex, insula, cingulate cortex, frontal operculum, superior temporal sulcus, and medial frontal gyrus (Hamdy et al., 1999; Martin et al., 1999), while others demonstrate patterns of hemispheric laterality in specific neural regions such as the insula, anterior cingulate cortex, parieto-occipital cortex, and superior temporal sulcus (Martin et al., 2001; Mosier et al., 1999). Furthermore, MEG and TMS studies have reported temporal shifts in hemispheric lateralization with the left hemisphere preferentially activating during the more voluntary oral phase of swallowing followed by an increase in right hemisphere activity during the more reflexive pharyngeal phase of swallowing (Dweizas et al., 2003; Teismann, Dweizas, Steinstraeter, \& Pantev, 2009). Given such discrepancies between functional imaging swallowing studies, the cortical representation of swallowing remains a ripe area for investigation.

In recent attempts to address the inconsistent reports of hemispheric laterality and regions of cortical activity during swallowing, researchers focused on the differences between voluntary vs. reflexively triggered swallows. For example, Kern and colleagues (2001) compared the cortical activity that occurred when individuals were given cues to voluntarily swallow to the cortical activity during swallows that were triggered reflexively by injecting boluses of water into the pharynx (2001). The results showed bilateral activity in the primary motor and somatosensory cortices during both swallowing conditions; however, the voluntary swallows elicited additional activity in the 
prefontal cortex, cingulate cortex, parieto-occipital regions, and insula (Kern et al., 2001). Alternatively, other studies have used a variety of swallow-related motor control tasks to understand better the functional role of cortical activity during the different phases of swallowing. Swallow-related motor tasks are simple movements such as tongue-tapping, tongue tip elevation, lip puckering are simple motor tasks that mimic individual movements performed at some point during swallowing (Robbins et al., 2008). For example, Martin et al. (2001) were among the first to contrast cortical areas of activity during swallowing and tongue movements and found that the regions of cortical activity did not differ between tasks. However, the strength of activity significantly varied according to task with the left pericentral and anterior parietal cortex, rostral anterior cingulate cortex, and right operculum/insula activating more during swallowing while the anterior cingulate, supplementary motor area, premotor, primary motor, and somatosensory cortices on the right activated more during tongue movement (Martin et al., 2001). In a study designed to dissociate the oral components from the pharyngeal components of swallowing, Malandraki et al. (2009) used three motor control tasks: a "preparing to swallow" task, a tongue tip tapping task, and a throat clearing task, to identify shared areas of neural activity between the tasks and swallowing. The results revealed shared patterns of bilateral premotor, primary sensorimotor and parietal cortex activity during both swallowing and tongue tapping with stronger activity occurring during the tongue tapping (Malandraki et al., 2009). In contrast, the throat clearing task induced stronger patterns of activity in the insula when compared to swallowing, and the "prepare to swallow" tasks did not activate any regions more so than other tasks. Together, the imaging studies provide evidence that different neural regions provide very specific functional contributions to the physiological movements performed during the oral and pharyngeal phases of swallowing. However, additional information regarding the time course of swallowing neurophysiology is needed to understand better the way that the functional dynamics of cortical activity differentially contribute to the oral versus pharyngeal phases.

\section{Sensorimotor Integration Theory}

As with other complex motor tasks involving muscles of the oral cavity, pharynx, and larynx, swallowing relies heavily upon a cooperative interplay between sensory and motor regions in the brain. Motor areas are responsible for planning and executing swallowing movements, while sensory areas predict the outcomes of movements, provide on-line adjustments for movements, and provide feedback from previously executed movements (Humbert 2011). Coordination of cortical sensory and motor contributions to swallowing is achieved through the process of 'sensorimotor integration' (SMI) (Hamdy et al., 1999; Miller, 2002; Zald \& Pardo, 1999). SMI is defined as the neurophysiological process that detects, associates, and integrates multimodal sensory information, derived from the body and/or environment, which determines, elicits, and shapes on-line motor behavior (Ayers, 1972; 1989).

According the Kandal, Schwartz, \& Jessell (2000), there are three basic principles that govern SMI: 1) sensory information is processed in a serial relay via multiple 
parallel pathways running from peripheral sensory receptors to the primary sensory cortices and on to uni-modal association cortices; 2) sensory information is organized in the sensory association cortices to yield an 'integrated' representation of multimodal sensory information; and 3) multimodal sensory information is relayed to frontal motor planning areas to prepare for action. In addition, SMI incorporates cognitive associations, based on experience to adapt and improve future performances of movement (Ayers, 1989; Bear, Conners, \& Paradiso, 2007; McNeil, 2009). In other words, SMI combines sensory information with motor commands to prepare the system to act, mediate action execution, and interpret feedback that facilitates adaptive adjustments in behavior via associative learning processes (Wolpert, 1999).

The neurophysiology of SMI differs according to particular movements that are required to perform an action and the resulting sensory experiences. However, three common patterns of 'higher-order' SMI must occur prior to action execution (Kandal et al., 2000). First, the posterior association areas, located within the temporal, parietal, and occipital lobes, process sensory information that is specific to a particular modality such as audition, vision, or somatosensation (Bear, Connors, \& Paradiso, 2007). Second, the limbic association areas provide situational context, allowing the brain to associate sensorimotor experiences with emotions and long term memories (Kendal, 2000). And third, anterior association areas in the pre-frontal cortex provide motor plans that prepare the system to execute the desired motor movement (Kandal et al., 2000).

Many theoretical models attempt to explain the functional role of SMI during action execution. Recently, the concept of 'feed-forward' or 'predictive internal models' has become one of the more predominantly explored and accepted theories used to explain motor skill acquisition, coordination, and control. Internal models are defined as sensorimotor associations that are formed through functional coupling between motor plans from previously executed motor actions and the resulting sensory experiences of those actions (Kawato, 1999). More specifically, as motor commands are sent to the peripheral muscles, an internal model, i.e. efference copy of the motor commands to perform a movement and the predicted sensory consequences of the movement, is sent from the PMC to somatosensory regions where sensory feedback from movements is interpreted (Wolpert \& Miall, 1999). When a mismatch is detected between the sensory predictions and the sensory experience, online motor adjustments are made to correct movement errors (Wolpert, Ghahramani, \& Flanagan, 2001). Functionally, the sensory predictions of internal models allow the brain to: 1) compensate for temporal delays in receiving and interpreting sensory feedback, 2) estimate the outcome, or sensory consequences of actions, and 3) enable on-line monitoring and corrections for erroneous motor commands (Miall \& Wolpert, 1999). Thus, anticipating the sensory outcomes of a movement enables the performance of smooth coordinated actions without total reliance upon sensory feedback. Given that swallowing requires the rapid performance of a complex series of movements with extraordinary temporal and sequential acuity, it seems logical to posit that feed-forward internal models mediate SMI during swallowing.

The importance of SMI contributions to swallowing is often discussed, but the way in which SMI mediates swallowing performance remains largely unknown. For 
example, the state of lingual movements as the bolus moves from the oral cavity to the pharynx issues the "go-ahead" to send motor commands that transition the food or liquid from the pharynx to the esophagus. The predicted sensory results of pharyngeal and esophageal bolus transport are the safe transition of the food or liquid to the upper gastrointestinal (GI) tract. Based upon this sensory prediction, the vocal folds abduct to re-open the airway as the pharyngeal structures return to a resting state and the esophageal sphincters begin transitioning the bolus to the upper GI tract. However, if a mismatch occurs between the expected sensory consequences and sensory feedback during pharyngeal bolus transport, adjustments in the strength of the pharyngeal swallow and the duration of vocal fold adduction would occur in an attempt to prevent aspiration of the food or liquid into the airway.

Recently, feed-forward internal models were proposed to explain the way in which sensory and motor regions coordinate with one another to control the voluntary oral phase of swallowing as well as support the 'central pattern generator' (Jean, 1984), i.e. reflexive control of pharyngeal swallowing (see Leopold \& Daniels , 2010 for a full review). Assuming that oropharyngeal swallowing behavior is represented and mediated by forward modeling, questions regarding the neuroanatomical and neurophysiological control of internal models require attention. The neuroanatomy of internal models remains uncertain. Researchers suggest that the cerebellum $(\mathrm{Cb})$ is the primary mechanism responsible for creating, storing, and updating internal models for sensorimotor experiences (Wolpert, Miall \& Kawato, 1998; Shadmehr \& Krakauer, 2008; Zuccaro, 2013). The Cb plays a role in the creation and maintenance of internal models and is understood by looking at the neurophysiology of the $\mathrm{Cb}$, as well as the functional pathways that connect the $\mathrm{Cb}$ with cortical sensory and motor areas. The $\mathrm{Cb}$ is known to coordinate balance, postural adjustments, and complex voluntary movements (Nolte, 1999). The multifaceted nature of $\mathrm{Cb}$ contributions to complex movement depends upon an intricate network of the neural circuitry connecting the $\mathrm{Cb}$ with the cerebral cortex, the brain stem, and the spinal cord (Nolte, 1999). Cb circuitry consists of cerebello-cortical layers, deep nuclei, and Cerebellar afferent and efferent fibers (Bhatnagar, 2002; Nolte, 1999).

Functionally, the $\mathrm{Cb}$ mediates balance and eye movement via the vestibulecerebellum pathway, while the spino-cerebellum and cerebro-cerebellum pathways provide information to mediate voluntary movement by developing feed-forward internal models of motor skills (Kandal, 2000; Wolpert et al., 1999). The sophisticated sensorimotor feedback circuitry of the $\mathrm{Cb}$ is primarily regulated by cortico-cerebellum pathways that receive cortical sensory information via the middle cerebral peduncle, and in response sends corrective motor information via ventrolateral thalamic projections to the PMC and primary motor cortices, thus forming a functional cortico-cerebellar sensorimotor feedback loop (Kandal, 2000). However, not all information traveling from the $\mathrm{Cb}$ to the cortex is responsive in nature; the $\mathrm{Cb}$ also is thought to be involved in the planning and rehearsal of movement (Wolpert et al., 1999). Evidence from both neural models and lesion studies support the notion that the $\mathrm{Cb}$ provides anticipatory, as well as on-line motor, corrections to facilitate coordinated movement in a continuous fashion (Bear et al., 2007; Kendal, 2000; McNeil, 2009). 
Although the $\mathrm{Cb}$ is considered the main neuroanatomical underpinning of internal models, the development and maintenance of these experience-driven behavioral representations requires a widely distributed sensorimotor network. Ito (2008) provides one the most comprehensive neurophysiological internal models: 1) the premotor cortex, supplementary motor area and anterior cingulate cortex coordinate to plan and sequence movements according to the efference copy of motor commands from the internal model; 2 ) the primary motor cortex sends motor commands to control the peripheral structures (i.e. muscles) during movement; 3 ) sensory feedback is transmitted from the primary somatosensory cortex; 4) the motor efferent copy of movement is updated and sent back to the $\mathrm{Cb}$ for updates; 5) the $\mathrm{Cb}$ sends the updated internal model to the PMC and primary sensorimotor cortices for on-line error detection and correction (2008).

The central representation of swallowing and the proposed neuroanatomy of forward internal models involve the same cortical sensory and motor regions. As mentioned, swallowing studies show that PMC activates prior to swallowing which prepares the system to act. It is also well known that the primary motor cortex controls the online execution of swallowing movements and regulates the adaptive qualities of movement based upon sensory feedback from the somatosensory cortex and the posterior sensory association areas. Thus, continuous motor activity in the PMC and the primary motor cortex coupled with incoming sensory feedback from the primary somatosensory cortex may represent the principal mechanism of SMI during swallowing.

\section{EEG Analysis of Sensorimotor Activity}

Cortical patterns of sensory and motor activity have traditionally been analyzed using fMRI and PET imaging techniques; however, the temporal resolution of these techniques is poor. Given that the performance of swallowing requires such a high degree of temporal acuity and is classified, diagnosed, and treating according to which temporal phase is affected, understanding the time-course of cortical SMI during swallowing is critical. EEG offers one possible solution to the problem of mapping the temporal patterns of SMI during swallowing. Using EEG techniques, event-related dynamics of neural activity are represented by increases or decreases in the 'power' that are mapped across time. (Pfurtshceller \& Silva, 1999). Increases or decreases in the spectral power of frequency bands directly correlate with increases or decreases in the degree of synchronization exhibited by a particular neuronal population (Neuper \& Pfurtscheller, 2001). Increases in spectral power are thought to correspond to 'inactive' or 'inhibited' cortical regions, as well as muscle activity, while decreases in spectral power are thought to correspond to regions of cortical activity (Neuper \& Pfurtscheller, 2001). Thus, event-related increases in the spectral power of frequency bands reflect the event-related synchrony (ERS) or 'inactive' patterns of neural activity or muscle activity, and decreases in the spectral power of frequency bands reflects a pattern of event-related desynchronization (ERD), which corresponds with active cortical regions (Neuper \& Pfurscheller, 2001). 
Oscillatory patterns of neural activity have been divided historically into five different 'rhythms' or frequency bands: delta ( 1-4 Hz), theta $(\sim 4-8 \mathrm{~Hz})$, alpha $(\sim 8-12$ $\mathrm{Hz})$, beta $(\sim 13-25 \mathrm{~Hz})$, and gamma $(\sim 35-100 \mathrm{~Hz})$ that differentially activate during the performance of cognitive, perceptual, and movement-based tasks. Recently, EEG and MEG studies aimed at analyzing SMI have begun to analyze the alpha and beta frequency bands in combination. The combined 'mu rhythm' is characterized by an initial spectral peak in the $\sim 10 \mathrm{~Hz}$ 'alpha' frequency band and a second spectral peak in the $\sim 20 \mathrm{~Hz}$ 'beta' frequency band, considered a global measure of cortical sensorimotor activity (Chen, Bin, Daly, Gao, 2013; Hari, 2006; Jensen, Goel, Kopell, Pohja, Hari \& Ermentrout, 2005; McGarry, Russo, Schalles \& Pineda, 2012; Pineda, 2005; Pineda, 2008). As with other rhythms, mu rhythm activity is measured via time-locked decreases and increases in the signal amplitude reflecting 'suppression' (i.e. neural activity) and 'enhancement' (i.e. neural inhibition or neural idling) respectively (Neuper and Pfrurtscheller, 2001; Pfurtshceller \& Silva, 1999).

Electrophysiological studies using whole-head EEG and MEG imaging techniques have reported mu rhythm suppression during tasks that require SMI. For example, a variety of visual and auditory perception studies provide evidence of mu rhythm suppression while viewing (Ruther, Brown, Klep, Bellebaum, 2014), hearing (Pineda et al., 2013), and imagining or virtually experiencing (Wagner, Solis-Escalante, Scherer, Neuper, \& Muller-Putz, 2014) the performance of actions. The alpha frequency band is known to be involved in sensory perception and has been found to suppress in response to auditory and visual stimuli (Capotoso, Bablioni, Romani, \& Corbetta, 2009; Freunberger et al., 2008; Maclin et al., 2011). Additionally, several studies have reported mu-alpha band suppression in response to tactile stimulation applied to the fingers (Ai \& Ro, 2014) and hands (van Ede, de Lange, Jensen, \& Maris, 2011), which has been localized to the primary somatosensory cortexand is thought to mediate perceptual somatosensation, in general. Alternatively, the beta frequency band is known to provide a general measure of motor activity (Kilavik, Zaepffel, Brovelli, MacKay, \& Riehle, 2013; Neuper \& Pfurtscheller, 1996; Stancak et al., 2000; Muller et al., 2003). In studies of cued movement performance, beta band suppression has been measured in the PMC prior to the onset of movement, and is thought to reflect the efference motor copy of movements (Kilavik et al., 2013; Pineda et al., 2013; Wagner et al., 2014). Beta suppression within the primary sensory and motor cortices has been shown during the performance of finger and thumb (Tsai, Jung, Chien, Savostyanov, \& Makeig, 2014), hand and foot (Hermes et al., 2010; Neuper, Wortz, \& Pfurtscheller, 2006; Yuan et al., 2009), walking and running (Lau et al., 2014; Wagner et al., 2014), tongue (Morash, Bai, Furlani, Lin, \& Hallett, 2008), and swallowing movements (Morash et al., 2008; Suntrup et al., 2014). Therefore, it seems reasonable to predict that EEG measured patterns of mu rhythm activity will provide cortical measures of SMI during swallowing.

\section{EEG: Independent Component Analysis}

Although EEG offers an excellent opportunity to analyze the temporal aspects of SMI during swallowing, the spatial resolution of EEG has been traditionally viewed as 
poor. This is because EEG surface electrodes record electrical activity from multiple electrical fields generated internally (i.e. neural electrical potentials) as well as externally (i.e. non-neural electrical potentials) (Pfurtscheller, 1996). Extracranial electrical potentials typically reflect muscular artifacts as well as other forms of incidental electrical noise (Onton, Westerfield, Townsend, \& Makeig, 2006). This is further complicated by 'volume conduction' in which electrophysiological signals project linearly to nearly every scalp electrode in the array (Onton et al., 2006). Thus, EEG susceptibility to muscle artifacts and the effects of volume conduction has limited the employment of EEG neuroimaging techniques in the past. However, recent advancements in blind source separation techniques have improved the spatial sensitivity of EEG, providing a valid means to analyze the spatial and temporal characteristics of cortical SMI during swallowing.

Independent component analysis (ICA) has been shown to separate and sort EEG signals into temporally independent and spatially fixed functional components (Onton et al., 2006). Using an 'information processing' blind source separation technique, ICA applies an algorithm to raw signal data that temporally sorts the data into an 'un-mixed matrix' (Onton et al., 2006). Once generated, the 'unmixed matrix' is multiplied by the number of raw EEG channels to yield a 'weighted' spatial representation of independent components (Maekig \& Onton 2008; Onton et al., 2006). Subsequently, the 'mixing matrix' or inverse of the 'unmixing matrix, is multiplied by the 'unmixing matrix' to obtain the amplitude values of the activity exhibited by each IC, allowing the forward projection of EEG activity to be traced back from the scalp map to the most likely cortical dipole source of activity (Onton et al., 2006). In other words, IC scalp maps are generated based upon a forward model that projects the spatial distribution of electrical potentials recorded from the scalp (Onton et al., 2006). Subsequently, inverse source modeling may be applied to IC scalp map distributions, which calculate the most likely equivalent cortical dipole (ECD) source of cortical activity (Schimpf, Ramon, Haueisen, 2002; Onton et al., 2006)

Using ICA to localize cortical sources of SMI, several EEG studies have localized mu components during the perception or imagination of movement to the premotor and/or primary sensorimotor cortices (Bowers et al., 2013; Chuang, Ko, Jung, \& Lin, 2014; Makeig et al., 2004; Naeem, Brunner, Leeb, Graimann, \& Pfurscheller, 2006; Tsai, Jung, Chien, Savostyanov, \& Makeig, 2014). In addition to providing a novel means to localize patterns of SMI, measured via mu components across groups and conditions, ICA has been suggested as a potential solution to problems that muscle artifacts pose to EEG signals during studies involving movement (Ma, Tao, Bayram, Svetnik, 2012; van der Meer, Tijssen, Bour, Rootselaar, Nederveen, 2010). Recently, a number of studies have used ICA to separate muscular components from neural components, including studies of tongue movements (Morash, Bai, Furlani, Lin, Hallet, 2009), finger movements and hand movements (Morash et al., 2009; Shou et al., 2012), walking and running movements (Gwin et al., 2010; Wagner et al., 2012), knee and ankle movements (Gwin and Ferris, 2012), and foot movements (Morash et al., 2009). Collectively, the studies above all reported mu-alpha and mu-beta ERD in component clusters that were localized to the premotor and/or primary motor and somatosensory cortices during action 
execution, and further validated the use of whole head EEG and ICA to analyze dynamic changes in SMI during tasks that require movement.

Although ICA has been shown to address muscle artifacts during movement, the methodological application of ICA to EEG data containing artifacts varied from study to study. To address this issue, McMenamin et al. (2011) proposed the following procedures to facilitate ICA attempts to 'unmix' neural (EEG) signals and non-neural (EMG) signals: 1) employ a minimum of 64 electrodes to capture whole-head EEG data; 2) simultaneously analyze all related conditions; and 3) visually inspect and manually extract all EMG components from the neural component clusters. According to several neurocomputational studies conducted by McMenamin and colleages, the techniques described above adequately resolve issues of overestimating or underestimating muscular artifacts for several reasons. First, using 64 electrodes reliably exceeded the Bayesian estimate of how many components were necessary (2012). Further, using 64 electrodes prevented ICA from 'leaking' muscle artifact data into neural components (McMenamin et al., 2012). Second, performing ICA on all related conditions limited the threat of overestimating muscle artifacts by providing an adequate sample (i.e. representation) of artifacts thereby supporting ICA abilities to estimate the spatial source of the signals (Onton et al., in press). Third, the slight variability associated with executing movements repeatedly may lessen ICA sensitivity to muscle artifacts that are not stereotypical in nature and as a result, careful inspection by 'expert human raters' is recommended to improve the sensitivity and specificity of using ICA to separate neural activity from muscle activity (Mcmenamin et al., 2012). Given the number of recent studies reporting the efficacy of using ICA to address muscular artifacts generated by large muscle groups that mediate gross movements, it seems reasonable to suggest that following the protocol described above, ICA will successfully separate swallow and tongue related muscle artifacts from cortical SMI during swallowing and tongue tapping tasks.

\section{Using EEG to Analyze Changes in Regional Neural Activity Across Time}

Patterns of ERS/ERD can be analyzed using various techniques; however, eventrelated spectral perturbations (ERSP) provide a means to visually analyze the temporally dynamic patterns of ERS/ERD in frequency bands across time that are time-locked to a particular event (Onton et al., 2006; Pfurtscheller \& Silva, 1999). Generating a colorcoded time-frequency graphic, ERSP depicting the average changes in spectral power between the baseline time period and the experimental condition time period are plotted (Makeig \& Onton, 2008; Makeig, Debener, Onton, \& Delorme, 2004). Further, ERSP provide a method for comparing and contrasting changes in ERS/ERD across subjects and conditions based upon IC scalp map and power spectra data (Makeig et al., 2004). Hence ERSP can be used to compare and contrast dynamic changes in SMI, reflected by clustered mu rhythm IC's exhibiting spectral peaks at $\sim 10 \mathrm{~Hz}$ (mu-alpha) and $\sim 20 \mathrm{~Hz}$ (mu-beta) across swallowing perception and swallowing performance experimental conditions. 


\section{Mu Cluster Activity During Different Phases of Swallowing}

One problem with using EEG to measure the neural activity during swallowing relates to the convoluted nature of physiologically separating the end of the oral phase from the beginning of the pharyngeal phase. To address this issue, Neuroscan EEG systems include two bipolar surface EMG electrodes, which can be placed over a particular muscle group to identify muscle activity during movement. The following muscle complexes are known to provide physiological references for analyzing muscular activity during swallowing: the orbicularis oris muscles represent the oral phase; the masseter muscles represent the oral phase; the submental muscle group represent oral and pharyngeal phases (Perlman \& Schulze-Delrieu, 1998; Vaiman et al., 2004); the infrahyoid muscle group represent the pharyngeal and esophageal phases (Balata et al, 2012; Ding, Larson, Logemann, \& Rademaker, 2002; Ertekin et al., 1997; Spiro \& Gay, 2004; Vaiman, Gabriel, Eviatar, Segal, 2005; Vaiman \& Eviatar, 2011). While electrophysiological data obtained from the submental muscle group measures muscular activity during both the oral and pharyngeal phases (Ding et al., 2002), the infrahyoid muscle group allows for interpretation of pharyngeal phase activity that is dissociated from the oral phase. Thus, ERSP analysis of the infrahyoid muscle component provides a temporal reference for interpreting patterns of ERS/ERD in mu components across the time-course of swallowing.

\section{Linking Perception and Production}

Sensorimotor integration provides a foundation for multidimensional associations between motor acts, sensory experiences, and the internal (i.e. cognitive) representations of actions (Vasant \& Hamdy, 2013). Theorists such as Hickok \& Hauser (2013) argue that mirror neurons represent 'sensorimotor association cells' that selectively respond to observed actions for sole the purpose of expanding or refining one's cognitive representation (i.e. internal model) of a particular motor skill. Others suggest that mirror neurons provide a functional link between action observation and action execution. Mirror neurons, first discovered in the F5 area of macaque monkey (Rizzolatti et al., 1996), activate when the monkeys perform an action and passively view the same action performed by another monkey (Rizzolatti et al., 1996; Rizzolatti \& Craighero, 2004). In humans, Brodmann's area 44, located within the inferior frontal gyrus (IFG), and the rostral area of the inferior parietal lobule (IPL) are considered homologous structures to the frontal (area F5) and parietal (areas PF, PFG, and PG of the inferior parietal cortex) mirror neuron regions of the macaque monkey (Buccino et al., 2001; Chong, Cunnington, Kanwisher, \& Mattingly, 2008; Molengerghs, Cunnington, \& Mattingley, 2012; Rizzolatti \& Craighero, 2004; Rozzi, Ferrari, Bonini, Rizzolatti, \& Fogassi, 2008). Although, the premotor cortex and inferior parietal lobule are considered the 'classic' components of the mirror neuron system, the following additional neural regions reliably activate during action observation-execution experimental paradigms: the dorsal premotor cortex, the superior parietal lobule, the posterior regions of the medial temporal gyrus, the cerebellum, the superior temporal sulcus, the anterior cingulate cortex, and the 
insula (Molenberghs et al., 2012). Evidence of mirror neuron activity in regions outside of the classical premotor cortex and inferior parietal lobe regions support the notion of a diverse SMI network to support higher order processing of motor commands and sensory experiences related to the performance of complex movements.

A number of EEG studies have reported mirror neuron activity, measured via mu suppression, in response to observing actions such as pointing (Avanzini et al., 2012), clenching or gripping (Braadbart et al., 2013; Mathukaswamy et al., 2004), foot movement (Neuper, Wortz, Pfurtscheller, 2006), and speaking (Crawcour, Bowers, Harkrider, Saltuklaroglu, 2009), which is generally considered a 'downstream' measure of SMI emanating from cortical sensory and motor regions (Pineda, 2005; 2013).

Recently, questions regarding the localization of mu suppression were explored by Arnstein et al. (2011) who simultaneously employed fMRI and EEG to measure SMI during the observation and imitation of hand actions, and found mu suppression correlated with increases in BOLD signals emanating from the premotor cortex, primary somatosensory cortex, and the inferior parietal lobule which regions. Another study conducted by Braadbaart \& colleagues (2013) analyzed the relationship between mu rhythm suppression and BOLD signals during the observation and imitation of hand actions, and also reported similar findings of a strong negative correlation between $\mathrm{mu}$ suppression and BOLD activity streaming from cortical sensory and motor regions in the frontal and parietal lobes (Braadbaart et al., 2013). Taken together, these studies provide strong evidence that mu suppression provides a valid index of 'higher order' SMI, and suggest a functional link between the observation and imagination of action execution. Given that mu suppression has been found in response to perceiving a variety of biologically relevant actions, it seems logical to anticipate mu suppression in response to the observation of swallowing.

Motor skill acquisition and mastery is achieved through repetitious exposure to multi-modal learning experiences (Kolb, Boyatzis, \& Mainemelis, 1999). In other words, interacting with the environment, observing and imitating the actions of others and repeatedly practicing actions collectively drives motor skill learning (Lahav, Saltzman, Schlaug, 2007; Paz, Wise, \& Vaadia, 2004; van der Helden, van Schie, \& Rombouts, 2010). Some authors argue that the perception-production link is critical to motor skill learning (Arbib et all., 2000 Iacoboni, 2009; Rizziolatte, Fogassi, \& Gallese, 2001). However, the role of SMI during the perception, acquisition, and mastery of motor skills remains unclear. As such, the overarching goal of the study is to identify the spatial and temporal dynamics of sensorimotor contributions to the performance and the perception of normal human swallowing behavior. In doing so, results may help researchers better understand 1) phase specific functional contributions of sensorimotor integration during oropharyngeal swallowing, and 2) the functional link between the performance and the perception of normal swallowing behavior. In order to accomplish this goal, this study addresses three aims:

- Aim 1: Identify sensorimotor and muscular activity during swallowing using EEG and ICA. Aim 1 Hypotheses predict that the source of cortical sensorimotor components found during swallowing will be localized to 
$\mathrm{PMC} /$ sensorimotor areas, and that the muscular infrahyoid EMG components will be identified via ERS spread across all frequencies ranging from $7-30 \mathrm{~Hz}$ during the pharyngeal and esophageal phases of swallowing.

- Aim 2: Measure changes in sensorimotor activity across the oral and pharyngeal phases of swallowing using ERSP. Aim 2 Hypothesis predict that time-frequency analyses of mu components will reveal patterns of mu rhythm ERS and ERD, which will be interpreted as feedforward / feedback measures of SMI during swallowing. Interpretations will be discussed further in relation to the time-course of infrahyoid muscle activity separating the pharyngeal phase of swallowing from the oral phase of swallowing.

- Aim 3: Descriptively compare the patterns of sensorimotor activity that occur during the performance of swallowing with patterns of sensorimotor activity during the perception of swallowing. Aim 3 Hypotheses predict that both the perception of swallowing and the performance of swallowing will reveal mu rhythm ERD; however, the mu ERD during the performance of swallowing is expected to demonstrate greater power as compared with the perception condition. 


\section{CHAPTER 3. METHODOLOGY}

\section{Participants}

Twenty-five normal adults, 20 females and 5 males with a mean age of 29 years old and no reported history of cognitive, communication, or swallowing impairments participated in this study. Participants were recruited from the University of Tennessee and the greater Knox County community. According to the Edinburgh Handedness Inventory, all study participants were right handed with the exception of one ambidextrous participant (Oldfield, 1979). The study protocol was approved by the University of Tennessee Health Science Center IRB board, and all participants gave their informed consent to participate in the study prior to beginning the experiment.

\section{Procedure}

All experimental data were collected in a double walled sound proof audio booth fit with a faraday cage to minimize electromagnetic interference. During EEG data collection, participants were seated comfortably in a reclining chair, and were given frequent rest breaks between experimental blocks to prevent fatigue. During data collection, participants were asked to avoid blinking and remain as still as possible to minimize the effects of muscular artifacts generated by extraneous movements. Two perceptual conditions and two performance conditions were included in this study. During the perceptual conditions, participants were instructed to attend to series of short video clips as they appeared on the screen. During performance conditions participants were instructed to remain as still as possible while waiting for a visual cue to begin moving according to the instructions provided before beginning each experimental condition block. All visual stimuli appeared on a 50-inch flat screen television located approximately 3 feet in front of the participant, and were presented via Neurobehavioral Systems (NBS) Presentation software (2014). Each experimental condition consisted of 8 blocks of ten trials, yielding a total of 80 trials per condition. The order of block presentation was randomized for each participant.

\section{EEG and EMG Data Acquisition}

EEG data were recorded using Compumedics NeuroScan Scan 4.5 software and the Synamps 2 system. Electrode impedances never exceeded $15 \mathrm{k} \Omega$ before, during, and after testing. Data were filtered $0.15-100 \mathrm{~Hz}$ and digitized via a 24-bit analog-to-digital converter at a sampling rate of $500 \mathrm{~Hz}$. Sixty-eight electrode channels were used to acquire whole-head EEG data, and were arranged according to the extended international 10-20 standard method using an unlinked, sintered NeuroScan Quik Cap (Towle et al., 1993). Electrodes were referenced to the common linked left (M1) and right (M2) mastoids. The electrooculogram (EOG) recorded eye movements using two VEOG electrodes placed superior and inferior to the left eye and two HEOG electrodes placed 
lateral and medial to the left eye. In an attempt to identify the time course of muscle activity generated during hyolaryngeal excursion, two bipolar surface electromyography (EMG) electrodes were placed over the infrahyoid muscle complex (Logemann, 1998; Perlman, Palmer, McCulluch \& Vandaele, 1999; Vaiman, Eviatar \& Segal, 2004; Vaiman $\&$ Nahlieli, 2009). Additionally, cardiac-related artifacts were recorded using two electrocardiography (ECG) electrodes placed over the right and left common carotid arteries.

\section{Experimental Conditions and Stimuli}

Experimental condition 1, the kaleidoscope perception task, was included in the study as the non-biological visual counterpart to experimental condition 2, the biologically relevant swallowing physiology perception task. The kaleidoscope video was created using the protocol published by Crawcour et al. (2009) using open source software obtained from the http://krazydad.com/kaleido/ website (2013). Similar to previous studies that investigated the effects of observing biologically relevant movements upon neural activity, a non-biologically relevant control task was created by scrambling the biologically relevant image (Crawcour et al., 2009; Grossman et al., 2000; Ulloa \& Pineda, 2007). In doing so, the perceptual qualities of the video are matched across conditions, with the only difference being whether or not a discernible pattern of biologically meaningful movement is perceived by participants (Ulloa \& Pineda, 2007). Accordingly, the current study took a still-frame image of oral and pharyngeal anatomy was taken from the animated swallowing video (experimental condition 2) and scrambled it to create the dynamic kaleidoscope video, Figure 1. During experimental condition 2, the swallowing perception task, participants viewed the "normal swallow physiology" video, purchased and downloaded from the Northern Speech Services website http://www.northernspeech.com/applications/ (2013). The "normal swallow physiology" video provided an animated demonstration of a normal swallow physiology lasting $\sim 3000$ ms (Northern Speech Services, 2013), Figure 2.

Experimental condition 3, the swallowing performance task, instructed participants to repeatedly self-administer a $5 \mathrm{ml}$ bolus of room temperature filtered water that was measured and delivered via a Provale dysphagia cup $\odot$. Participants were told to reach for the cup, accept a small sip of water, and hold the water in their mouth until viewing the visual cue to orally transition the bolus and trigger the pharyngeal swallow response. Experimental condition 4, the tongue taping performance task, instructed participants to repeatedly tap their tongue against the alveolar ridge. In accordance with the tongue tapping protocol described by Malandraki and colleagues (2010), participants were instructed to hold their jaw in a slightly open and somewhat relaxed position while they tapped the tip of their tongue to the roof of their mouth where the tops of the upper front teeth meet the roof of the mouth repeatedly at a comfortable rate. Tongue-tapping tasks are frequently used as control conditions in swallowing neuroimaging studies because physiologically tapping the tongue against the alveolar ridge mimics the tongue movements that transition the bolus from through the oral cavity during the 'oral transit phase' of swallowing (Humbert \& Robbins, 2007; Komisaruk et al., 2002; Malandraki, 

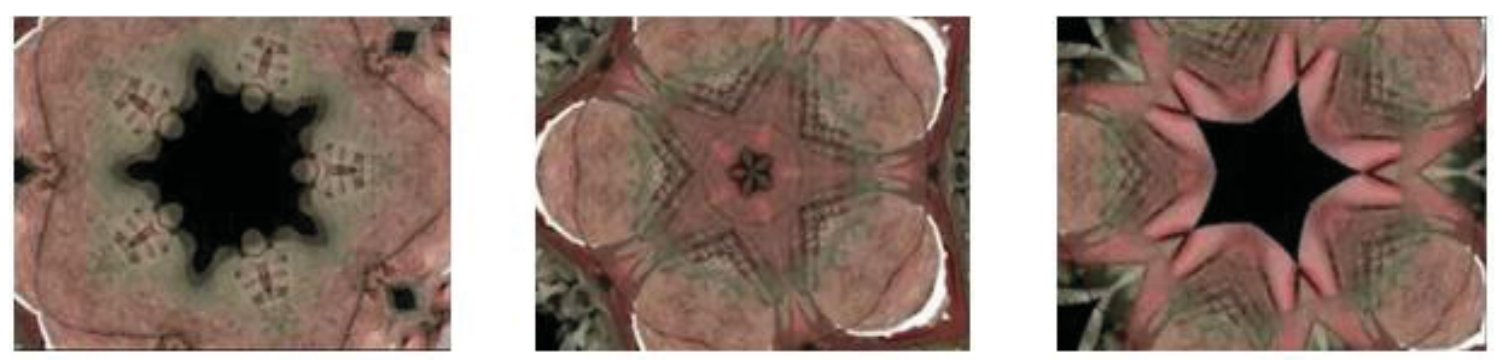

Figure 1. Still Frame Pictures Taken from the Kaleidoscope Video

Reprinted with permission from Northern Speech Services, MBSImP (C2013), http://www.northernspeech.com/applications/
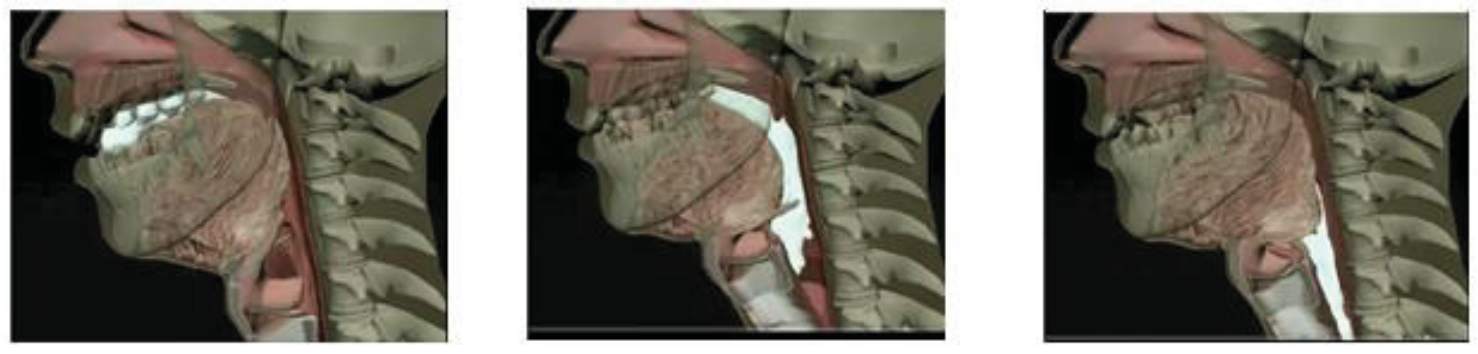

Figure 2. Still Frame Pictures Taken from the Animated Swallowing Video Reprinted with permission from Northern Speech Services, MBSImP (C2013), http://www.northernspeech.com/applications/ 
Sutton, Perlman, \& Karampinos, 2010; Martin et al., 2004). During both performance conditions, participants were instructed to wait until the visual cue to "go" appeared on the TV screen before performing the desired movement, i.e. swallowing or tongue tapping. For an overview of all conditions, see Table 1. In all four experimental conditions, $2000 \mathrm{~ms}$ inter-trial intervals (ITI) were inserted between individual trials. However, the timelines varied between the perception conditions and the performance conditions, because the performance conditions included an additional $7000 \mathrm{~ms}$ of silence inserted between the pre-stimulus baseline and the onset of the swallowing performance task to allow study participants to self-administer small sips of water via the Provale dysphagia cup $_{\odot}$ in the swallowing condition. In order to analyze the time-course of electrophysiological activity across both the perception andperformance conditions, the $7000 \mathrm{~ms}$ separating the pre-stimulus baselines from the onset of the performance tasks (i.e. swallowing and tongue tapping) were manually extracted from all trials yielding a common timeline that included a $1000 \mathrm{~ms}$ baseline followed by a $3000 \mathrm{~ms}$ experimental task (Figures 3-5).

\section{EEG Data Processing and Analysis}

Using EEGlab 12, which is an open source Matlab toolbox created by Delorme \& Makeig, 2004, raw EEG signal data were individually preprocessed and then concatenated across participants and conditions to undergo group processing.

\section{Individual Preprocessing and Analysis}

- Each raw data file was preprocessed separately for each condition

- The perceptual condition files were concatenated and ICA was applied

- The performance condition files were concatenated and ICA was applied

- All neural and non-neural IC's were analyzed to obtain dipole estimates of localization

\section{Group Processing and Analysis}

- Using the STUDY command in EEGlab, three separate modules (i.e. studies) were created to analyze: a) "in head" neural clusters during the perceptual conditions, b) "in head" neural clusters during the performance conditions, and c) "out of head" clusters during the performance conditions

- Principle component analysis (PCA) was performed to identify and cluster components that exhibited common spectral, topographic, and dipole attributes

- Left and Right hemisphere mu clusters were identified in the "in head" perceptual and performance studies

- The "in head" Mu clusters were localized using equivalent dipole models 
Table 1. Control and Experimental Conditions

Perception Tasks $\quad$ Performance Tasks

Kaleidoscope perception task $\quad$ Swallowing performance task

Swallowing perception task Tongue-tapping performance task

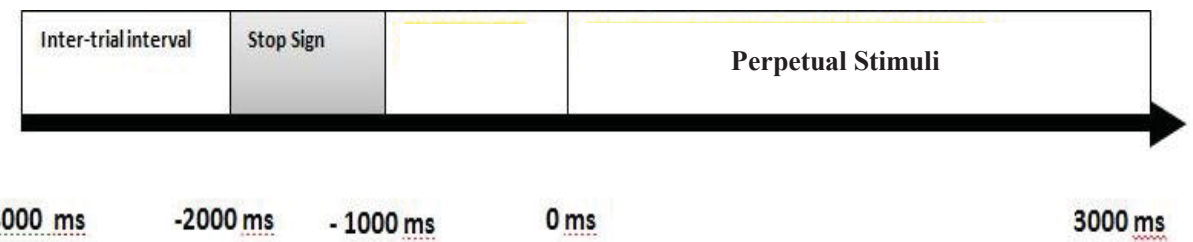

Figure 3. Timeline of Kaleidoscope and Swallowing Perception Condition

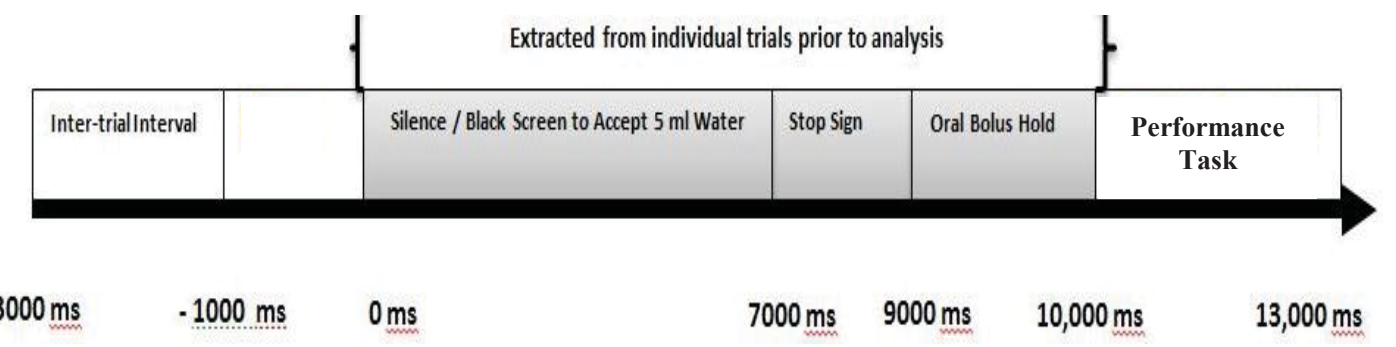

Figure 4. Timeline of Swallowing and Tongue-tapping

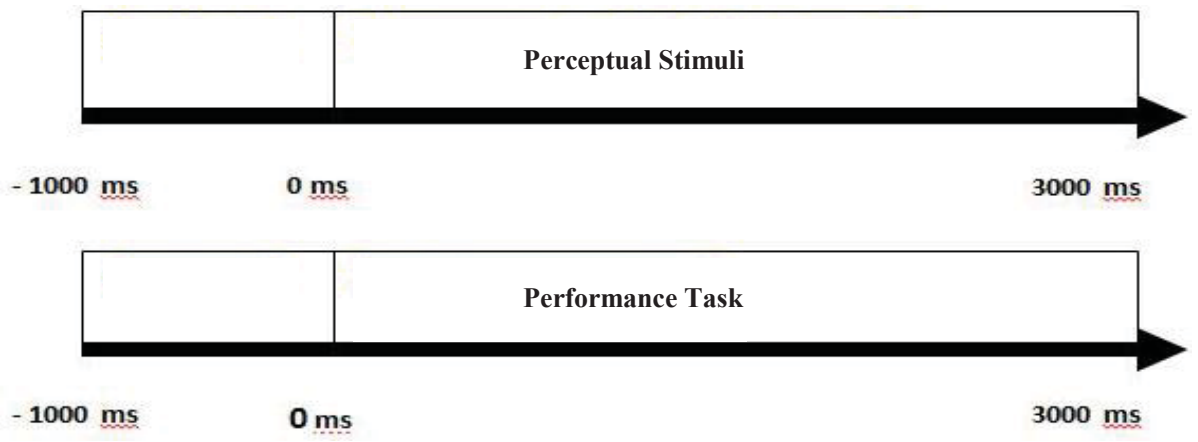

Figure 5. Epoched Timeline for All Four Perception and Performance Conditions 
- The "out of head" EMG component cluster was localized using equivalent dipole models

- Time-frequency analyses were performed on the mu clusters obtained from the perceptual and performance studies to analyze the time course of event related spectral perturbations

- Time-frequency analyses were performed on the "out of head" EMG component that represented EMG activity during swallowing

\section{Data Preprocessing and Analysis}

Using EEGlab, which is an open source Matlab toolbox (Delorme \& Makeig, 2004), whole-head EEG data were resampled at $256 \mathrm{~Hz}$, and channels were located using BESA head model coordinates. Data were re-referenced to the mastoids (M1 and M2), and were band passed filtered between 3 and $39 \mathrm{~Hz}$. Across all four conditions, individual trials were segmented or 'epoched' to begin $1000 \mathrm{~ms}$ prior to time point zero, which marked the beginning of the experimental task and ended at $3000 \mathrm{~ms}$. Thus, all data were epoched to begin at $-1000 \mathrm{~ms}$ and ended at $3000 \mathrm{~ms}$, yielding a sum of 4000 $\mathrm{ms}$ of data in each individual trial. A minimum of 50 epochs were retained and preprocessed for all experimental conditions across participants. Epochs that were contaminated with gross muscular artifacts not associated with the desired movement during the production tasks, i.e. swallowing and tongue tapping, were identified and removed from the dataset. Specifically, surface EMG data recorded from the infrahyoid musculature were identified via powerful ERS across all frequencies (7-30) and preserved in the EEG signal for the purpose temporally identifying and separating the pharyngeal phase of swallowing from the oral phase of swallowing (Vaimann et al., 2004; 2005; 2009).

Following EEG data preprocessing, i.e. re-referencing, band pass filtering, epoching, and rejecting artifacts, condition datasets were concatenated across subjects and ICA was applied to the raw EEG signal data (Onton, 2008; Makeig \& Onton, 2008) using the extended version of the 'runica' algorithm in the EEGLAB v12 ICA toolbox. In each participant, 66 independent components (ICs) were generated from a 68 channel electrode array across conditions by decomposing or 'unmixing' raw EEG signals into maximally temporally independent and spatially fixed components (Jung, 2000; Onton \& Makeig, 2006). Upon separating signals into functional independent components (IC's), spatially fixed projections of component activity were plotted on scalp maps representing the projected (i.e. forward model) distribution component activity (Delorme \& Makeig, 2004). After ICA was performed on all of the individual condition datasets, the perceptual conditions and the performance conditions were concatenated separately and then run through ICA to obtain maximally temporally independent and spatially fixed a) kaleidoscope perception + swallowing perception IC's, and b) swallowing performance + tongue tapping performance IC's for each participant.

After generating IC's from the concatenated perception and production condition data, "in head" equivalent current dipole (ECD) models localized the cortical sources of 
IC scalp map distributions in each of the 66 scalp maps generated by each participant. In addition, ECD models were generated for the "out of head" concatenated production IC scalp maps. Using the DIPFITx2 functions, ECD models were created by manually coregistering electrode channel locations with the Boundary Element Head Model. ECD's were only included in further group analysis if the "in head" ECD's explained more than $85 \%$ of the variance in the IC scalp map distributions, and "out of head" ECD's were retained if the dipole models explained more than $50 \%$ of the variance in the IC scalp map distributions. The variance in IC distributions was lowered to accommodate the increase in variance due to the muscle movement at the level of the infrahyoid muscle complex.

Upon obtaining the "in head" and "out of head" dipole estimates of the spatial IC scalp map projections for each participant across concatenated conditions, group analyses were performed using the STUDY toolbox in EEGLAB v12. For all three group analyses, component measures were 'pre-computed' based upon the following criteria: a) the degree of variability explained by each IC, b) the spectral or frequency characteristics of IC activity, and c) the scalp map spatial distribution of each IC (Makeig \& Onton, 2011). Next, a 'pre-clustering array' was built to instruct the subsequent principle component analyses (PCA) to cluster IC's demonstrating similar spectral, dipole, and scalp map characteristics across participants in both the "in head" and "out of head" group analyses using the 'k-means' procedure (Delorme \& Maekig, 2004). Across all studies, IC's found to exhibit topographic, spectral, or dipole characteristics more than 3 standard deviations from the mean were automatically excluded from clusters.

Following IC clustering, the IC's that were assigned to the right (R) and left (L) hemisphere 'anterior' mu clusters were manually inspected and validated based upon the following criteria: 1) topographic scalp maps exhibited a localized concentric pattern of signal distribution along the midline of the $\mathrm{L}$ and $\mathrm{R}$ hemispheres; 2) power spectra included spectral peaks in the mu-alpha $(\sim 10 \mathrm{~Hz})$ and mu-beta $(\sim 20 \mathrm{~Hz})$ ranges; 3$)$ dipole estimates reflected the "in head" spatial map of the signal distribution displayed in the cluster topographic scalp map; 4) ERSP displayed patterns of ERD in the mu-alpha $(\sim 10$ $\mathrm{Hz})$ and mu-beta $(\sim 20 \mathrm{~Hz})$ frequency bands; 5) scalp maps, power spectra, and ERSP exhibiting characteristics of overt EMG or myogenic activity were excluded from the cluster (see McMenamin et al., 2010 methods supplemental materials for more details; McMenamin et al., 2011). In addition, proximal component clusters were individually inspected to also ensure that all mu components were assigned correctly during the automated PCA clustering procedure.

In contrast, the "out of head" production conditions were inspected to identify the EMG component cluster found to best exemplify muscle activity associated with hyolaryngeal excursion during swallowing. The most exemplary EMG component cluster was chosen by inspecting and verifying that each of the IC's in the cluster exhibited the following signal properties that are known to be associated with myogenic activity: 1) topographic scalp map displays of diffuse signal distribution; 2) spectra with overwhelming patterns of high frequency activity; 3) dipole source estimates were clustered outside of, and inferior to, the head model; 4) ERSP displaying 
overwhelmingly powerful signal enhancement ( ERS) measured across all frequencies of interest (7-30 Hz) (McMenamin et al., 2010; 2011).

To analyze changes in the power of ERS/ERD expressed by the $\mathrm{R}$ and $\mathrm{L}$ anterior mu component clusters across time, ERSP were analyzed within the 7-30 Hz range. ERSP decomposing the time-frequency domains of the ICs retained in the mu component clusters were computed from Morlet sinusoidal wavelet transformations initially set at 3 cycles and rising linearly to 20 cycles at $40 \mathrm{~Hz}$ (Delorme \& Makeig, 2004). Of particular interest were comparisons of changes in ERS/ERD power of the mu-alpha $(\sim 8-13 \mathrm{~Hz})$ and mu-beta $(\sim 15-25 \mathrm{~Hz})$ frequency bands during experimental conditions, beginning at time point zero and ending at $3000 \mathrm{~ms}$, relative to their respective $1000 \mathrm{~ms}$ pre-stimuli baseline periods. ERSPs of the baseline period were computed from a pseudoset of timefrequency values that were randomly sampled 200 times from a sliding latency time window. To test for conditional effects between: 1) the kaleidoscope and swallowing perception conditions and 2) the swallowing and tongue tapping performance conditions, EEGLAB bootstrapping statistics were employed with an alpha value set at .05 (Manly, 2007; Sigh \& Xie, 2013). False discovery corrections (FDR) were applied due to multiple hypotheses (Bejamini \& Hochberg, 1995). 


\section{CHAPTER 4. RESULTS}

\section{Performance Conditions: Independent Component Clustering}

Component clustering of "in head" IC's across participants in the performance conditions (swallowing and tongue tapping) yielded 9 neural clusters demonstrating activity in the alpha $(8-13 \mathrm{~Hz})$ frequency range and/or the beta $(15-25 \mathrm{~Hz})$ frequency range. These clusters were localized to the $\mathrm{R}$ and $\mathrm{L}$ premotor cortices, the $\mathrm{R}$ superior temporal sulcus, the $\mathrm{R}$ and $\mathrm{L}$ anterior cingulate cortices, the $\mathrm{R}$ dorsal-posterior cingulate cortex, and the occipital lobes. For the purposes of this study, the anterior R and $\mathrm{L}$ 'sensorimotor' or mu component clusters were the only ones further analyzed and discussed. The R mu component contained 27 ICs from 19 or $76 \%$ of study participants and the L mu component contained 31 ICs from 16 or 64\% of study participants (Figures 6B and 7B) with both the $\mathrm{R}$ and $\mathrm{L}$ mu cluster spectra exhibiting stereotypical peaks at $\sim 10 \mathrm{~Hz}$ and $\sim 20 \mathrm{~Hz}$, respectively (Figures 6A and 7A). Further, both $\mathrm{R}$ and $\mathrm{L} \mathrm{mu}$ clusters yielded average equivalent cortical dipoles (ECD) localized to the $\mathrm{R}$ and $\mathrm{L}$ premotor cortices (BA 6) with $\mathrm{x}, \mathrm{y}, \mathrm{z}$ Taliarach coordinates expressed as [(R) 37, -9, 45] and $[(\mathrm{L})-35,-8,50]$ (Figures 6C and 7C). The percent of residual variance (RV) left unexplained by the dipole models was $11.4 \%$ for $\mathrm{R} \mathrm{mu}$ and $11.62 \% \mathrm{~L}$ mu clusters. In a separate analysis that was performed to cluster "out of head" IC' across participants in the performance conditions, a prominent cluster representing infrahyoid EMG was found. The infrahyoid cluster contained 22 IC's from 18 or $72 \%$ of study participants with ECD's located outside of, and inferior to the head model and $13.92 \%$ of the RV unexplained by the dipole model (see Figure 8).

\section{Performance Conditions: Event Related Spectral Perturbations (ERSP)}

ERSP time-frequency analyses measuring "in head" $\mathrm{R}$ and L mu clusters exhibiting were found to exhibit significant patterns of ERD when compared to baseline. ERSP results are visually displayed in a graphic with the first panel depicting swallowing ERSP, the second panel depicting tongue-tapping ERSP, and the third panel depicting significant differences $(\mathrm{pFRR}<.05)$ between the two conditions with significance represented in red (see Figure 8). Paired t-tests were performed to compare the "in head" swallowing and tongue tapping conditions and revealed significant differences ( $\mathrm{pFDR}<$ $.05)$ in ERD of the mu-alpha $(8-13 \mathrm{~Hz})$ frequency range and the mu-beta $(15-25 \mathrm{~Hz})$ frequency bands of the $\mathrm{R}$ mu cluster. In both conditions, mu ERD began $\sim 500 \mathrm{~ms}$ after time point 0 , when participants received the cue to swallow or begin tongue tapping. However, during swallowing it was observed that mu-alpha and mu-beta ERD was significantly stronger in the $\mathrm{R} \mathrm{mu}$ cluster beginning at $\sim 500 \mathrm{~ms}$ and continuing until 3000 $\mathrm{ms}$ when the epoch ended. Furthermore, the onset and offset of the most robust period of ERD in the R mu cluster coincided directly with the onset ( $\sim 800 \mathrm{~ms})$ and offset $(\sim 1700$ $\mathrm{ms}$ ) of "out of head" EMG infrahyoid activity, which was used to temporally demarcate the pharyngeal and esophageal phases of swallowing. In contrast to the $\mathrm{R}$ mu cluster, the "in head" L mu cluster did not reveal significant differences between the swallowing and 
A)

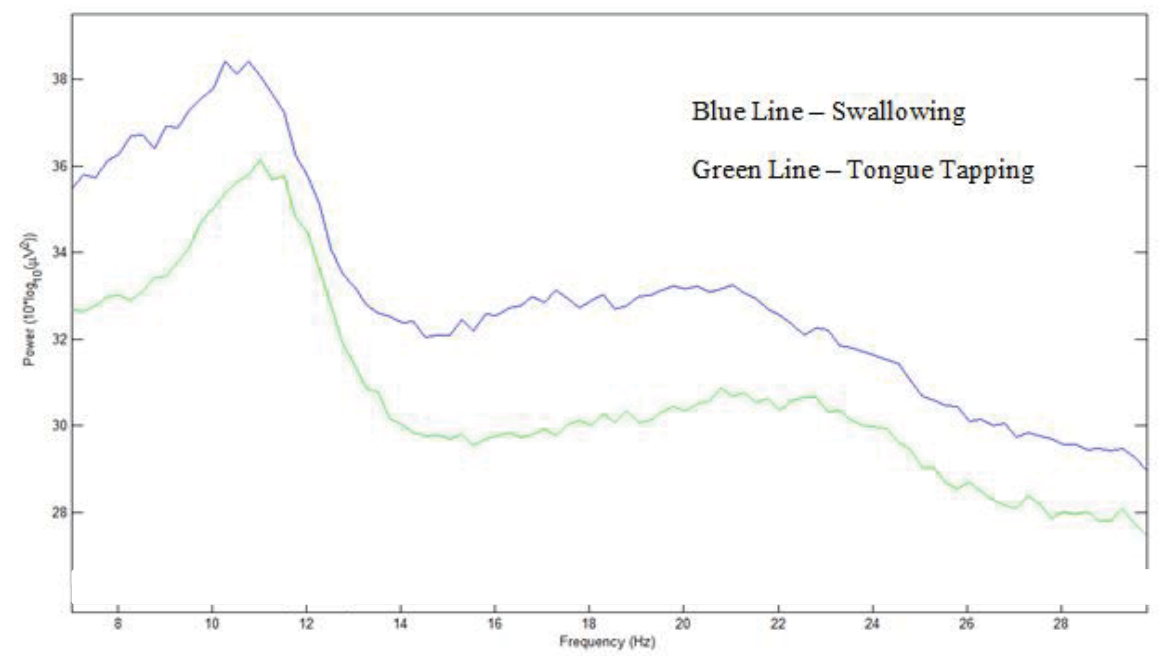

B)

C)
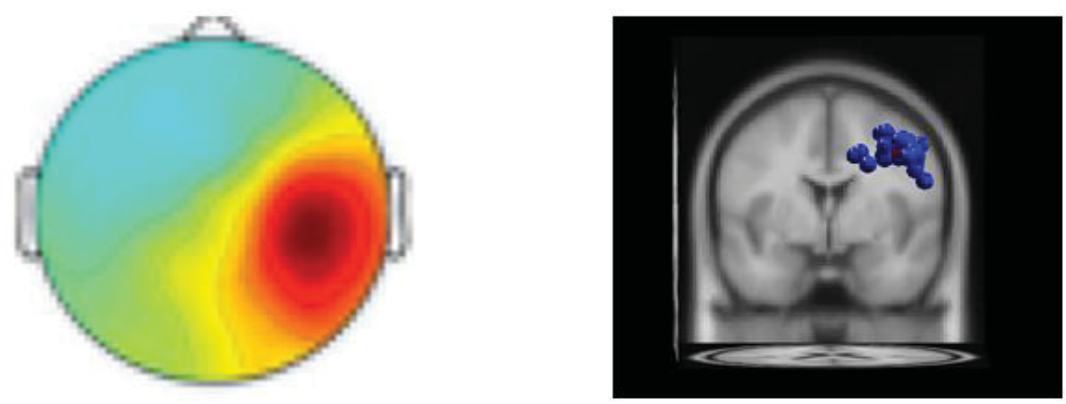

Figure 6. Right Mu Spectral Displays for Swallowing and Tongue-tapping (A), Right Mu Topographic Scalp Map Projections (B), Right Mu Cluster ECD's Located in the PMC (C) 
A)

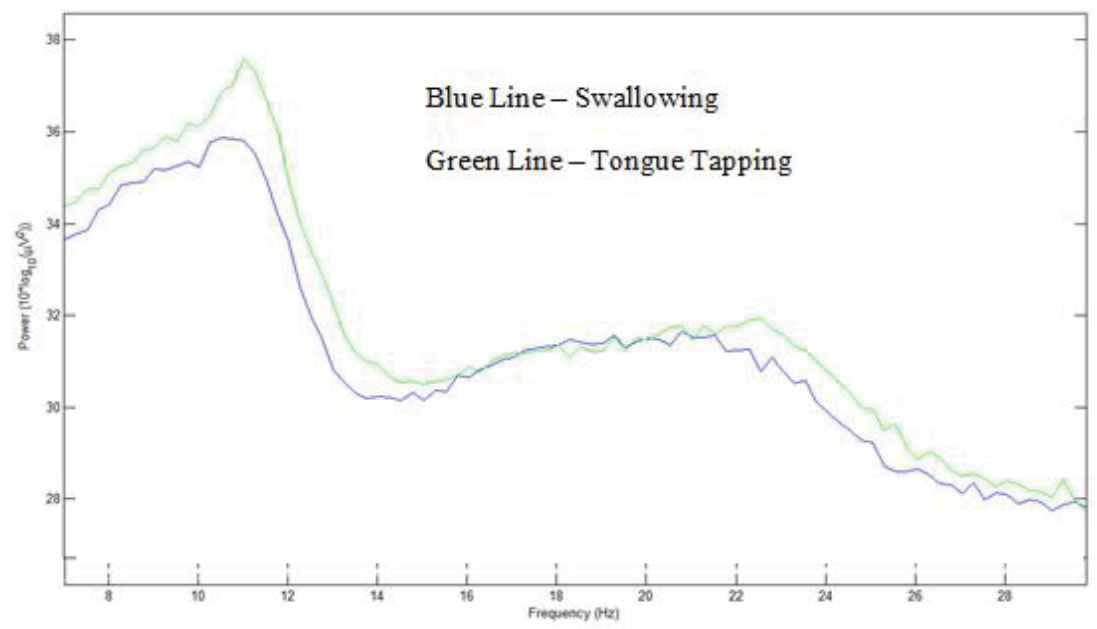

B)

C)
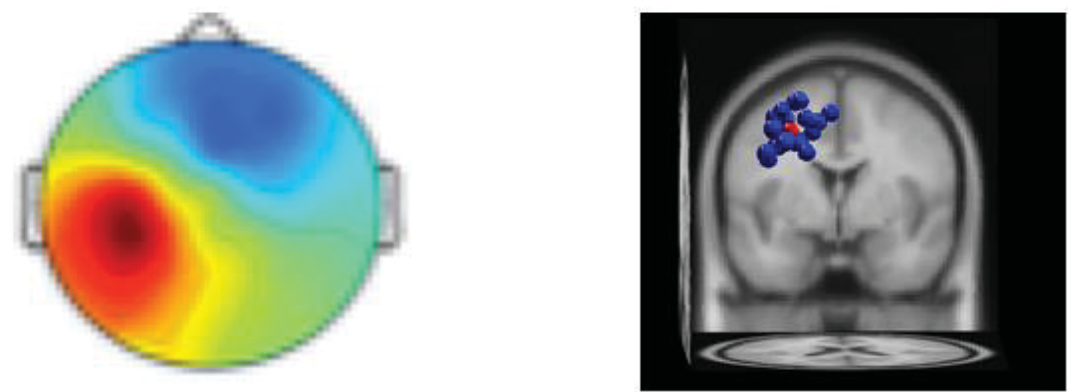

Figure 7. Left Mu Spectral Displays for Swallowing and Tongue-tapping (A), Left Mu Scalp Map Projections (B), Left Mu Cluster of ECD's Located in the PMC (C) 

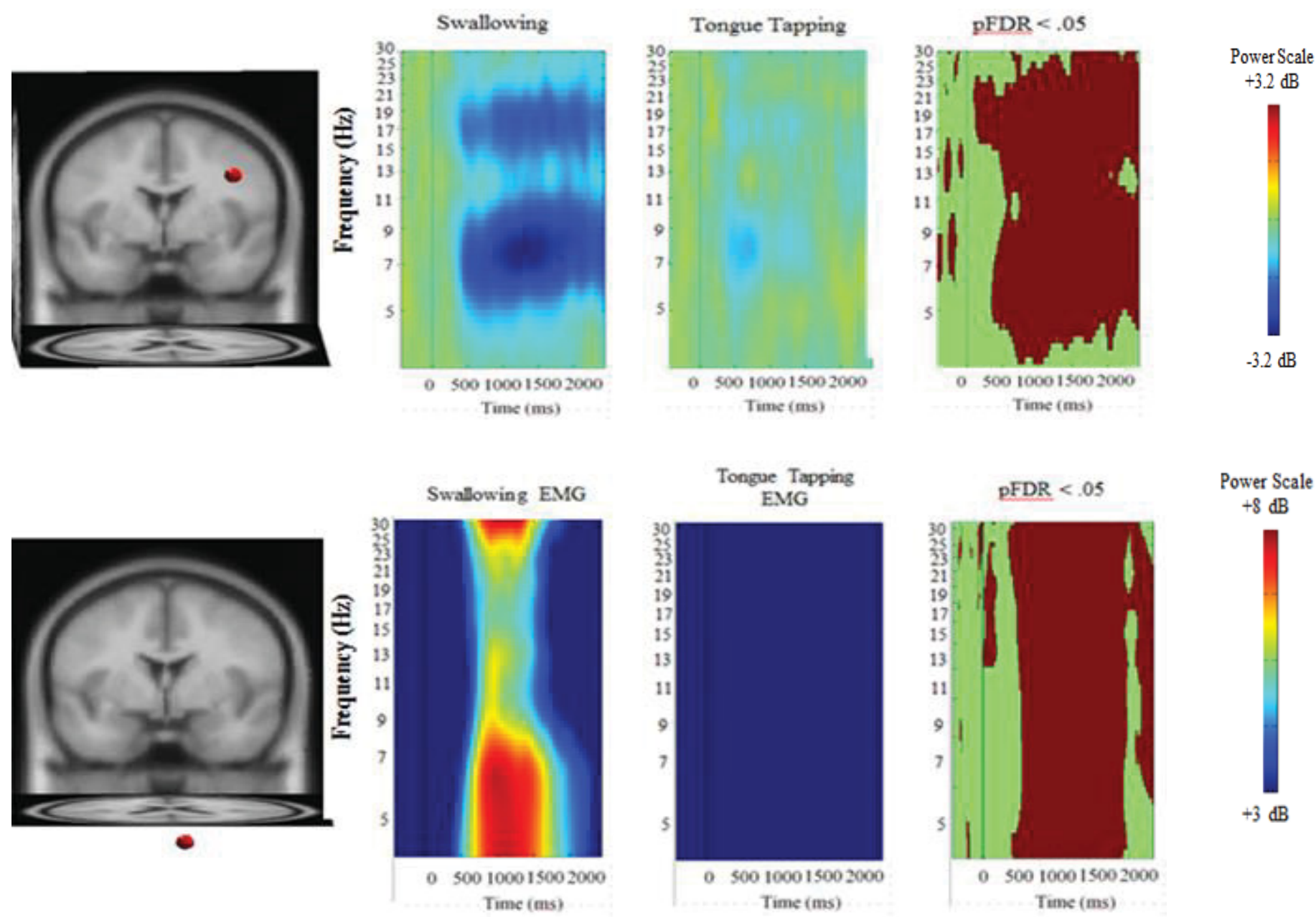

Figure 8. Right and Left Mu IC Cluster Average ECD's and ERSP alongside EMG Cluster ECD and ERSP 

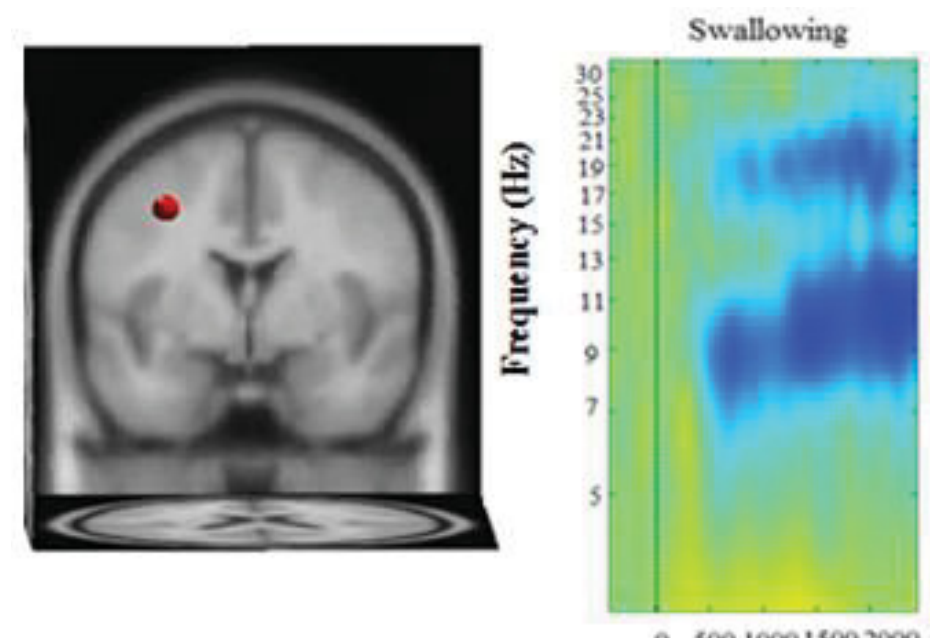

e 500100015002000 Time (ms)

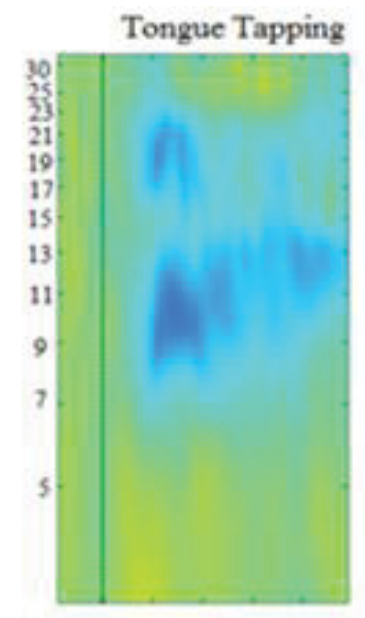

o 500100015002000 Time (ms)

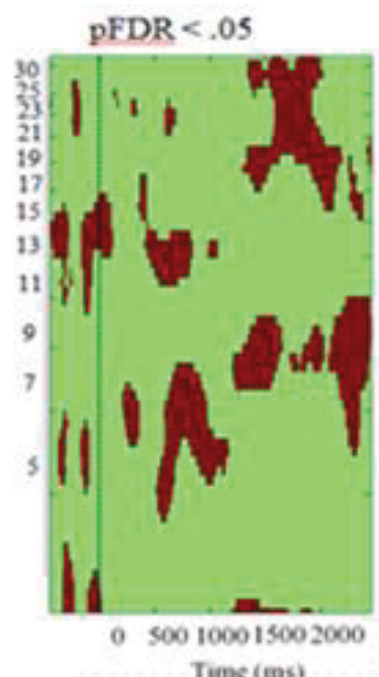

Time (ms)
Power Scale

$+3.2 \mathrm{~dB}$

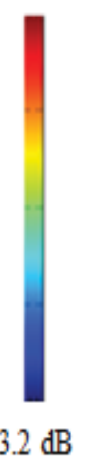

Figure 8. (Continued) 
tongue tapping conditions in the mu-alpha $(8-13 \mathrm{~Hz})$ frequency range or the mu-beta $(15-25 \mathrm{~Hz})$ frequency range. Similar to the R mu cluster, ERD in the L mu cluster began $\sim 500 \mathrm{~ms}$ after time point 0 and continued to $3000 \mathrm{~ms}$, marking the end of the epoch. Additionally, ERD in the $\mathrm{L}$ mu-beta $(15-25 \mathrm{~Hz})$ frequency range after $1000 \mathrm{~ms}$ notably weakens in the tongue tapping condition.

\section{Perception Conditions: Independent Component Clustering}

Clustering of the "in head" IC's across participants in the perception conditions (kaleidoscope and swallowing observation) yielded 12 neural clusters with activity in the alpha $(8-13 \mathrm{~Hz})$ frequency range and/or the beta $(15-25 \mathrm{~Hz})$ frequency range. These clusters were localized to the $\mathrm{R}$ and $\mathrm{L}$ middle frontal gyri, $\mathrm{R}$ and $\mathrm{L}$ anterior cingulate cortex, $\mathrm{R}$ superior temporal sulcus, $\mathrm{L}$ insula, and $\mathrm{R}$ and $\mathrm{L}$ parietal cortices. As with the analysis of the performance conditions, for the purpose of this study, the $\mathrm{R}$ and $\mathrm{L}$ anterior 'sensorimotor' or mu component clusters will be the only clusters further analyzed and discussed. The R mu cluster contained 30 ICs from 19 or $76 \%$ of study participants whereas the L mu cluster contained 25 ICs from 17 study participants or $68 \%$ of study participants (see Figures 9B and 10B). The spectral display of both mu clusters exhibited peaks at $\sim 10 \mathrm{~Hz}$ (mu-alpha) and $\sim 20 \mathrm{~Hz}$ (mu-beta) (Figures 9A and 10A). As seen in the performance conditions, both the R and $\mathrm{L}$ mu cluster average ECD were localized to the $\mathrm{R}$ and $\mathrm{L}$ premotor cortices and supplementary motor areas (BA 6) with $\mathrm{x}$, $\mathrm{y}, \mathrm{z}$ Taliarach coordinates expressed as [(R) 35, 2, 42] and [(L) -29, -3, 50] (Figures 11A and $12 \mathrm{~A}$, respectively). The percent of $\mathrm{RV}$ left unexplained by the dipole model was $11.2 \%$ and $12.09 \%$ for the $\mathrm{R}$ and $\mathrm{L}$ mu clusters respectively. 
A)

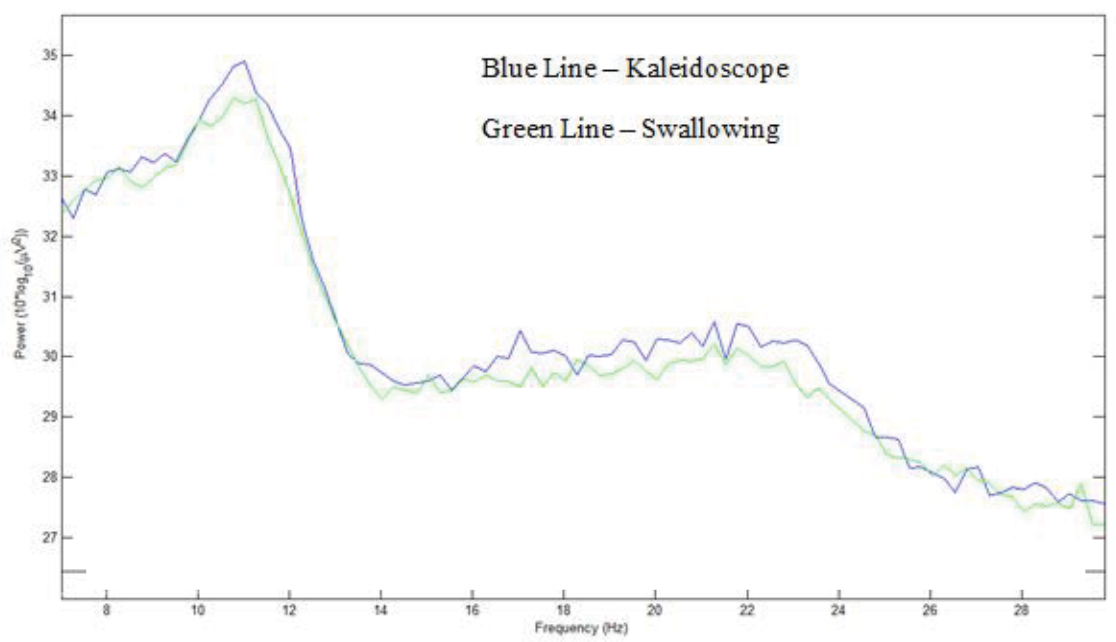

B)

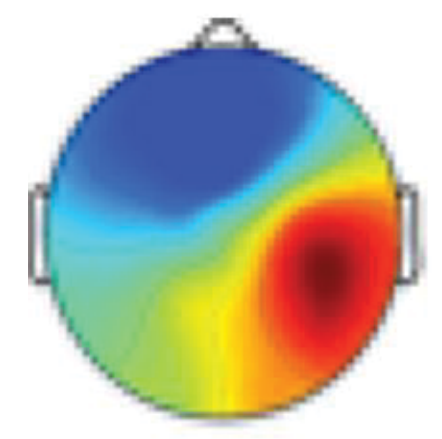

C)

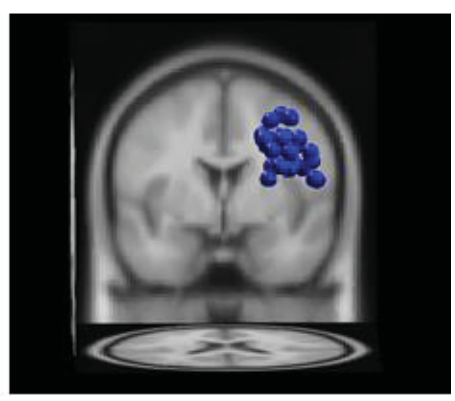

Figure 9. Right Mu Spectral Displays for Swallowing and Kaleidoscope Perceptual Tasks

(A), Left Mu Scalp Map Projections (B), Left Mu Cluster of ECD's Located in the PMC (C) 
A)

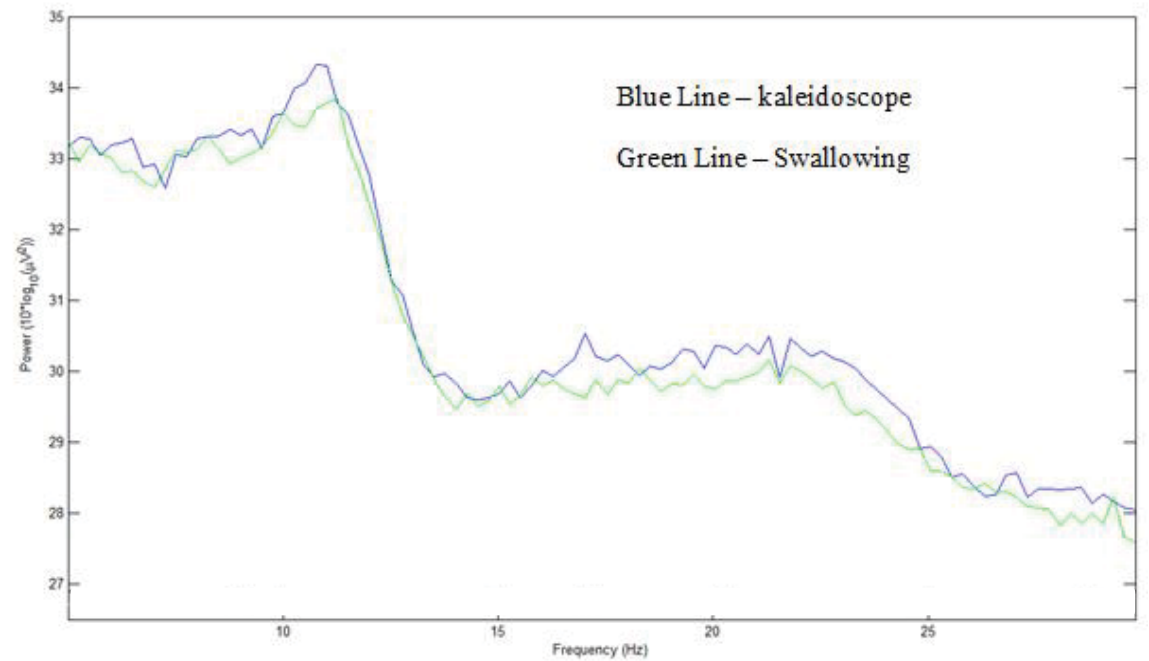

B)

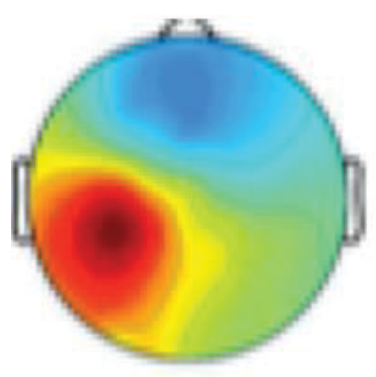

C)

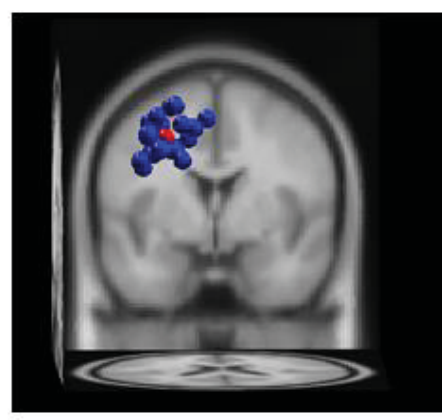

Figure 10. Left Mu Spectral Displays for Swallowing and Kaleidoscope Perceptual Tasks

(A), Left Mu Scalp Map Projections (B), Left Mu Cluster of ECD's Located in the PMC (C) 
A)

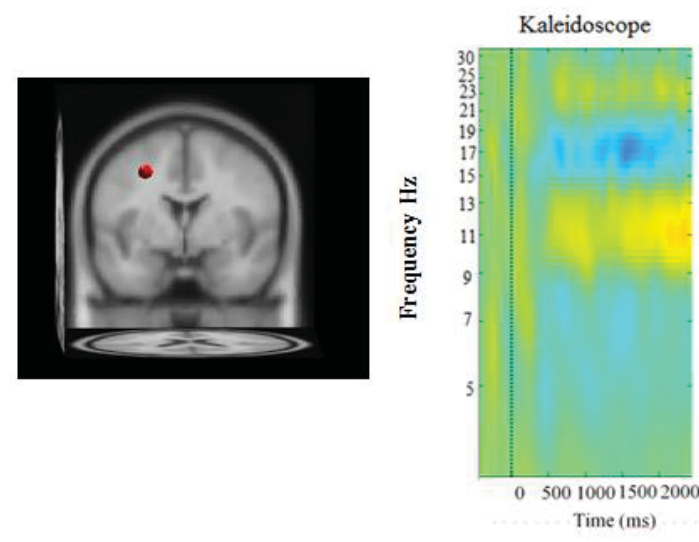

B)
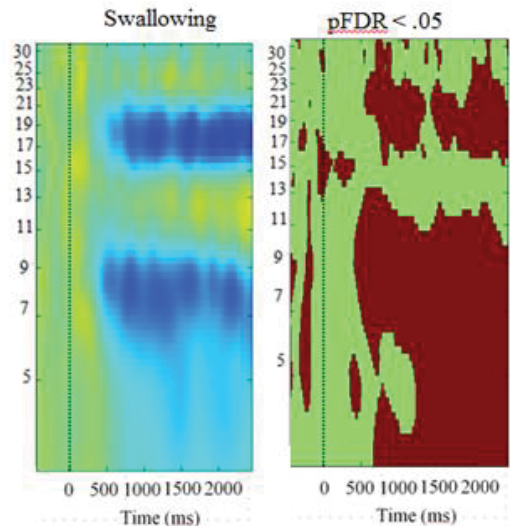

Power Scale $+3.2 \mathrm{~dB}$

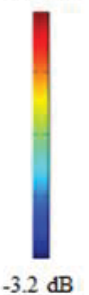

Figure 11. Left Mu IC Cluster Average ECD for Kaleidoscope and Swallowing Perceptual Tasks

(A), Left Mu ERSP on a Power Scale Ranging from $+3.2 \mathrm{~dB}$ to $-3.2 \mathrm{~dB}$ for Kaleidoscope and Swallowing Perception Conditions (B)

A)

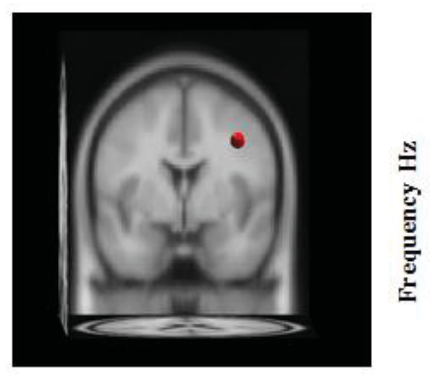

B)

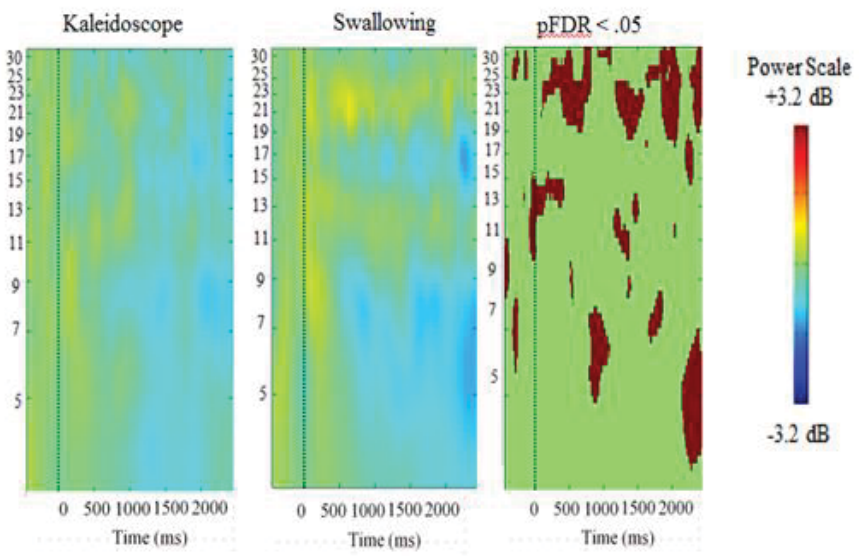

Figure 12. Right Mu IC Cluster Average ECD for Kaleidoscope and Swallowing Perceptual Tasks

(A), Right Mu ERSP on a Power Scale Ranging from $+3.2 \mathrm{~dB}$ to $-3.2 \mathrm{~dB}$ for Kaleidoscope and Swallowing Perception Conditions (B) 


\section{CHAPTER 5. DISCUSSION}

Three aims were addressed in this study. First, revealing bilateral mu component clusters via ICA and PCA demonstrated the sensitivity of this technique for identifying sensorimotor activity during the perception and performance of swallowing. Second, using time-frequency analysis techniques, ERSP revealed real-time changes in the amplitude (i.e. power) of mu-alpha and mu-beta ERD during the perception and performance of swallowing. Furthermore, time-frequency patterns of mu-alpha and beta ERD in relation to the onset and offset infrahyoid EMG activity, reflected by ERS spread across all frequency bands, provided a temporal reference for interpreting changes in the strength of sensorimotor activity during the oral vs. pharyngeal and esophageal phases of swallowing. Third, descriptively comparing patterns of mu-alpha and beta ERD during the perception of swallowing to patterns of mu-alpha and beta ERD during the performance of swallowing revealed similarities and differences in the strength and time course of sensorimotor activity during the perception (i.e. observation) and performance (i.e. execution) of swallowing. To our knowledge, this is the first study to use ICA and time-frequency analysis techniques to measure real time changes in cortical sensorimotor activity during swallowing. Thus, the results of this study provide a new lens through which to view the functional dynamics of cortical sensorimotor activity during the perception and performance of swallowing, and offer evidence to support future research into swallowing using this novel EEG technique.

According ECD source localization, bilateral mu clusters were localized to the PMC (BA 6) during the perception and performance of swallowing. This finding is consistent with numerous fMRI and PET swallowing studies that have shown activity in the PMC prior to, as well as during, the performance of swallowing (Kern et al., 201; Hamdy et al., 1999; Martin et al., 2001; Malandraki et al., 2009; Wantabe, Ishikawa, Yamada, \& Yamane, 2004). It also is consistent with recent EEG/ICA identifying mu components in speech perception and production. The PMC is adjacent to the primary motor cortex and is the control center in both feedforward and feedback control of complex motor sequences. (Houde and Nagarajan, 2011). Thus, when swallowing the PMC initiates motor commands (feedforward control). As the muscles move, sensory information from the movements is delivered to the PMC to help update and prepare subsequent movements. However, if motor control were to rely solely on this 'external' loop, the system would not be able to update itself quickly enough to make online corrections when necessary (i.e., an unexpected perturbation). In order to make online correction possible, as motor commands are delivered to the muscles, the PMC delivers an internal model (efference copy) of the expected sensory consequences of muscle movement to the somatosensory association region (posterior-inferior parietal lobe). The expected consequences can be compared to the movement goals in sensory regions before the true sensory information is provided from muscle movements, allowing sensory feedback to be delivered to the PMC quickly enough to allow online corrections. As the muscles begin to move, true sensory feedback is integrated into the loop and delivered to the PMC. The notion of the PMC being a sensorimotor hub is consistent with current findings. Mu components localized to the PMC (with activity likely spreading to the 
motor and sensorimotor cortex), were characterized by alpha and beta spectral peaks providing evidence of somatosensory and motor activity were found during swallowing. Therefore, changes in the spectral power of these peaks across time in these data (measured using ERSP) are currently interpreted as changes in sensory and motor activity. Additionally, similarities for the proposed role that action observation plays in activating an 'internal model' were found by investigating the patterns of mu-alpha and beta ERD during both perception and production of swallowing.

\section{Aim 1: Using ICA to Identify Sensorimotor and Muscle Activity}

The first aim of this study was to determine if applying ICA to whole head EEG data would allow for identification of cortical sensorimotor and muscular activity during the performance of swallowing. First, 76\% (19 of 25) study participants contributed IC's to the $\mathrm{R}$ mu component cluster and 64\% (18 of 25) study participants contributed IC's to the $\mathrm{L}$ mu component cluster with $\sim 10 \%$ of the residual variance left unexplained by the dipole model. The number of participants that contributed IC's to the $\mathrm{R}$ and $\mathrm{L} \mathrm{mu}$ clusters is consistent with the results of previous studies (Bowers et al., 2013; Gwin \& Ferris, 2011; Jenson et al., 2014; Makeig et al., 2004; Moore et al., 2012; Paek et al., 2014). Although the proportion of RV that was unexplained by the dipole model was $\sim 10 \%$ in both mu clusters, which is slightly higher than reports from previous studies, these findings are similar to those reported by Jenson and colleagues (2014). Considering that both studies included motor tasks involving movements of head and neck muscles, it seemed reasonable to conclude that the slight increase in RV was due to muscle activity generated during the performance conditions. Both the $\mathrm{R}$ and $\mathrm{L}$ mu clusters revealed average ECD that were localized to the PMC (BA 6), although it is likely that mu activity spread into neighboring motor (e.g., BA 4) and sensorimotor (BA 1,2,3) regions (see Jenson et al., 2014 and Bowers et al). The PMC and these adjacent regions play a critical roles in SMI, and have been consistently shown to activate during functional imaging studies of swallowing (Hamdy et al., 1999; Humbert and Robbins, 2007; Martin et al., 2004; Mosier \& Bereznaya, 2001; Suntrup et al., 2014).

In addition to finding mu clusters reflecting "in head" sensorimotor activity, a prominent "out of head" infrahyoid EMG component cluster from $72 \%$ of the particpants,. According to predictions, ICA identified and separated infrahyoid muscle activity from neural activity, enabling real-time analysis of changes in the power of SMI across temporally demarcated oral vs. pharyngeal phases of swallowing. A similar analysis was made by Jensen and colleagues (2014) in speech production. They reported EMG activity from periliablial regions as a marker of the speech initiation when producing words initiated with bilabial sounds. The current findings extend the utility of this technique to swallowing. Thus, finding independent mu rhythm and muscle component clusters during swallowing was seen as support for using ICA to measure real-time changes in cortical sensorimotor activity across physiologically distinct phases of swallowing. 


\section{Aim 2: Time-Frequency Analysis of Swallowing}

The second aim of this study was to use time-frequency analysis techniques to measure changes in sensorimotor activity across the oral and pharyngeal phases of swallowing. In support of aim 2 hypotheses and similar to MEG swallowing studies, the performance of swallowing elicited bilateral patterns of simultaneous mu-alpha and beta ERD, beginning at $\sim 500 \mathrm{~ms}$ into the trail and continuing until the trial terminated at 3000 ms (Furlong et al., 2004; Gow et al., 2004; Suntrup et al., 2014, Teismann et al., 2009; 2010; Wantabe et al., 2004). Mu rhythm ERD was reflected by decreases in the spectral power of the mu-alpha $(\sim 10 \mathrm{~Hz})$ and mu-beta $(\sim 20 \mathrm{~Hz})$ frequency bands (see figures 8 and 11 depicting blue bands of color that reflect ERD across time). The average latency from time point zero when the visual command to "go" (i.e. swallow) was delivered to the onset of mu ERD at $\sim 500 \mathrm{~ms}$ was consistent with response times reported in previous studies that have used a "go" signal to cue swallowing (Kern, 2001; Malandraki et al., 2009; Martin et al., 2001; 2004). Immediately following mu ERD at $500 \mathrm{~ms}$ into the trial, infrahyoid EMG activity, identified via strong ERS spread across all measured frequencies $(7-30 \mathrm{~Hz}$ ), was found to begin at $\sim 800 \mathrm{~ms}$ and end at $\sim 2300 \mathrm{~ms}$. Hence a close temporal relationship was observed between the time course of sensorimotor activity and the time course of muscle activity. Furthermore, the most robust period of mu-alpha and beta ERD directly coincided with the onset and offset of the strongest period of EMG activity. In terms of localization, both $\mathrm{R}$ and $\mathrm{L}$ mu clusters were localized to the PMC (BA 6), with the R mu cluster exhibiting more powerful (i.e. stronger) patterns of ERD than the L mu cluster.

\section{Mu-alpha and Mu-beta Across the Time Course of the Swallow}

As mentioned, the mu rhythm consists of two oscillatory peaks, one occurring at $\sim 10 \mathrm{~Hz}$ and the other at $\sim 20 \mathrm{~Hz}$ (Hari et al., 1998). Mu rhythm ERD, localized to the PMC and primary motor and somatosensory cortices has been shown during the performance of finger, hand, foot, and mouth movements (Hari \& Salmelin, 1997; Hari, 2006; Gazzola et al., 2006; Jenson et al., 2014). Hence the mu rhythm activity is generally thought to provide a functional measure of cortical SMI during movement execution. Beta activity (i.e., suppression) is widely known as a measure of motor activity, and similar to alpha ERD the functional properties of beta ERD are frequently interpreted based upon the timing and localization of activity. For example, beta ERD has been shown to be the most powerful during movement performance, and is considered a functional measure of ongoing motor commands to guide movement execution (Kilavik et al., 2013). Alternatively, beta ERD found prior to the initiation of movement during a variety of movement tasks, and is thought to underpin anticipating, planning, and preparing for the performance of intended movements (Kilavik et al., 2013). Several MEG studies have found beta ERD localized to the primary motor and somatosensory cortices as well as the PMC during swallowing, and have generally considered beta ERD a measure of cortical motor activity. However, to our knowledge, no EEG studies exist that have looked at patterns of beta ERD during swallowing. 
EEG studies involving finger, hand, foot, and tongue movements have shown beta ERD during the performance of simple repetitive movements (e.g. finger tapping or tongue tapping) (Gaetz et al., 2010; Morash et al., 2008). In the Jenson et al (2014) study, beta ERD was found in both the perception and production its speech, as well as in the time period prior to speech production. The authors interpreted these findings as evidence that mu beta ERD captures sensorimotor activity due to both actual muscle movements and to the generation of internal models used to drive real motor activity. While capturing this activity across time, they noted that by only looking at beta activity in the mu rhythm it was not possible to distinguish between these two sources of beta ERD during the time period of muscle activity. The patterns of beta ERD herein were similar to those found in speech production (Jenson et al., 2014). Therefore, it seems reasonable to interpret patterns of mu-alpha and beta ERD from a similar perspective. Thus beta ERD in swallowing is interpreted as a measure of cortical sensorimotor activity resulting from the generation of internal models and actual muscle movements.

Mu alpha ERD was pervasively observed alongside beta ERD in swallowing. Although alpha ERD has been found in response to tasks involving cognitive processes such as sustained attention and working memory (Klimesch, 2012), alpha has also been elicited during visual, auditory, and somatosensory perception tasks. Therefore, meaningful interpretations of alpha ERD must consider the particular context in which alpha activity was observed (Jenson et al., 2014). In addition, suppression of the alpha band of the mu rhythm is often interpreted as index of somatosensory activity. Therefore, considering the localization to the PMC, and the co-occurrence, of alpha suppression with beta suppression, it is logical to interpret alpha suppression as an index of sensory feedback to the PMC (Jenson et al., 2014). This interpretation is also consistent with the models of motor control discussed above. Depending on the time course relative to muscle movement, this feedback (i.e., alpha suppression) may result from the true sensory consequences of muscle movement and/or the earlier comparisons of the internal model to the expected sensory consequences. Together mu alph and mu beta ERD provide real time EEG evidence of a sensorimotor loops guiding the complex series of movements necessary to produce a swallow.

\section{Mu-alpha and Beta During the Oral vs. Pharyngeal and Esophageal Phases}

Based upon extant neuroimaging studies of non-swallow related movements such as tongue tapping, researchers have traditionally attributed PMC activity to sensorimotor processing demands associated with controlling tongue movement during the oral phase of swallowing. In contrast, the current study found stronger patterns mu-alpha and beta ERD, localized to the PMC, during the pharyngeal and esophageal phases of swallowing. One possible reason for finding less powerful patterns of mu ERD during the oral phase relates to the fact that the oral acceptance and bolus formation, i.e. the oral preparatory phase, was removed from all trials in order to exclude the gross muscular artifacts associated with hand and arm movements during self-administration of water via cup. As a result, only the 'oral transit' phase, whereby the bolus traveled posteriorly to trigger the pharyngeal swallow, remained in the trials. In other words, the initial muscle movements 
and corresponding sensorimotor activity occurred prior to the onset of data that was included in the swallowing performance trials, which began as participants were sitting still and holding water in their mouths waiting for the "go" cue to swallow. However, given the overwhelming increase in the power of mu-alpha and beta ERD during the pharyngeal and esophageal stages of swallowing, it seems more logical to conclude that the later phases of swallowing required a larger degree of cortical sensorimotor control than previously suggested.

Although, current views tend to emphasize the role of the PMC during the oral phase of swallowing, a seminal fMRI study of swallowing by Hamdy and colleagues (1999) found the strongest patterns of BOLD activity in the PMC during the pharyngeal and esophageal phases of swallowing. As a result the authors concluded that PMC is primarily responsible for planning and modulating muscle movements during pharyngoesophageal swallowing. This interpretation contradicted traditional views that considered the pharyngeal phase of swallowing to be primarily mediated by brainstem reflexes. Cortical sensorimotor contributions to the pharyngeal and esophageal phases of swallowing has also been argued in a series of TMS studies that applied repetitive transcranial stimulation (rTMS) to the R and L PMC and observed bilateral EMG activity in the skeletal pharyngeal and esophageal musculature, demonstrating a direct pathway from the PMC to pharyngo-esophageal muscle effectors (Aziz, Rothwell, Hamdy, Barlow, \& Thompson, 1996; Hamdy et al., 1998). In terms of laterality, the applying rTMS to the R PMC elicited the strongest responses from the pharyngeal and esophageal muscles, indicating a pattern of $\mathrm{R}$ hemisphere dominance (Hamdy, Aziz, Thompson, \& Rothwell, 2001; Aziz, Rothwell, Hamdy, Barlow, Thompson, 1996; Hamdy et al., 1996). Similar to previous fMRI studies by Hamdy and colleagues, the current study found stronger patterns of mu-alpha and beta ERD, localized to the PMC, during the pharyngeal and esophageal phase of swallowing. In addition, greater power (i.e. strength) was exhibited in R mu ERD when compared to L mu ERD across swallowing, and particularly during the pharyngeal and esophageal phases. Thus finding strong patterns of mu ERD across swallowing, and more specifically during the pharyngeal and esophageal phases, suggests that integrated feedforward and feedback sensorimotor processing in the PMC modulates the timing and strength of swallowing muscle movements, particularly during later pharyngeal and esophageal phases of swallowing. in nature, and emphasizes the potential for using behavioral sensory and motor driven swallowing interventions to stimulate swallowing neuroplasticity following neurological injuries impede function.

\section{Sensorimotor Integration in Swallowing vs. Tongue Tapping}

Mu-alpha and beta ERD followed the same general pattern of activity in the tongue-tapping task as the swallowing task., a finding which is consistent with reports from previous MEG, fMRI, and PET swallowing studies that have included tongue tapping as a control task to compare with swallowing (Malandraki et al., 2009; Martin et al., 2004; Peck, Haupage, Branski, Holodny, \& Kraus, 2009). However, the ERSP analyses using a conservative statistical approach revealed weaker bilateral alpha and 
beta ERD in the tongue-tapping task. This finding differs from other studies in which swallowing and tongue-tapping has been shown to elicit similar levels of PMC activity (Kern et al., 2001; Martin et al., 2001; 2004; Malandraki et al., 2009). However, these studies employed tongue tapping as a non-swallowing motor control task that elicits similar spatial patterns of neural activity, specifically in the PMC and primary motor and somatosensory cortices (Humbert \& Robbins, 2007; Malandraki et al., 2009). To our knowledge, tongue tapping has not been analyzed using ICA to localize the cortical source of activity, nor has the strength of activity been measured across time during swallowing and tongue tapping in this paradigm before. Upon using this novel technique to compare and contrast the strength of sensorimotor activity during swallowing and tongue tapping, differences in ERD strength between these two motor tasks indicates that swallowing is a more complex than tongue tapping because the sequence of motor activity during swallowing requires a greater degree of sensorimotor control. Weaker patterns of sensorimotor activity in the PMC and primary sensorimotor cortices has been shown in studies aimed at contrasting simple vs. complex finger movements and unimanual vs. bimanual hand movements (Kuhtz-Buschbeck et al., 2003; Nair, Purcott, Fuchs, Steinberg, \& Kelso, 2003). These findings continue to support the use of the ICA / ERSP technique for measuring sensorimotor control in motor tasks, as well as using tongue-tapping as a less complex control in swallowing studies.

\section{Aim 3: Descriptive Comparison of Swallowing Perception and Performance}

Diverging from the first two performance-driven aims of this study, the third aim was to compare descriptively sensorimotor activity during the performance of swallowing with changes in sensorimotor activity during the perception of swallowing. A number of EEG studies have reported suppression of power in the mu rhythm during the observation of a variety of biologically relevant actions such as grasping and gripping (Muthukumaraswamy et al., 2004; Perry \& Bentin, 2009; Streltsova, Berchio, Gallese, Umilta, 2010); ripping sheets of paper (McGarry et al., 2012); finger tapping (Cochin, Barthelemy, Roux, \& Martineau, 2008) speaking (Muthukaswamy, Johnson, Gaetz, \& Cheyne, 2006), and drinking (Kawai et al., 2009; Ushioda et al., 2012). In many of these studies, this activity, i.e. sensory stimulation resulting in sensorimotor activity, has been interpreted as a downstream measure of mirror neuron activation. However, it should be noted that many of these studies typically only included the 1 alpha frequencies in their definition of the mu (Muthuswamy et al., 2004; McGarry et al., 2012; Ulloa \& Pineda, 2007). While mirror neuron based theories can certainly be considered for the current data, given that the production findings were interpreted from the standpoint of sensorimotor control, the perception findings will be interpreted similarly.

Seventy-six percent or 19 of 25 study participants contributing IC's to the R mu component cluster and $68 \%$, or 17 of 25 study participants contributing IC's to the L mu component cluster. These findings are consistent with others and continue to indicate that the mu rhythm can be find robustly when measuring in perception tasks (Bowers et al., 2013; Jenson et al., Marshall, Young, \& Meltzoff, 2011; 2014; Pineda, 2013). The number of participants that contributed IC's to R and L mu clusters obtained from the 
perceptual conditions was almost identical to the number of participants that contributed IC's to mu clusters obtained from the performance conditions. Similar to the performance conditions, the R and L mu clusters were localized to the PMC (BA 6) $\sim 10 \%$ of the residual variance was left unexplained by the dipole model, and supported the hypothesis that the localization of mu-alpha and beta ERD would be the same across the perception and performance of swallowing

\section{Mu-alpha and Mu-beta During Perception: Swallowing vs. Kaleidoscope}

The ERSP analysis showed that $\mathrm{L}$ mu alpha and beta ERD co-occurred while watching swallowing (i.e., followed the same pattern as when participants actually swallowed). The onset of mu-alpha and beta ERD occurred simultaneously, beginning at $\sim 500 \mathrm{~ms}$ post stimuli onset, which again was noted to be consistent with reported response times in studies of mu ERD during the observation of biologically relevant actions (Avanziini et al., 2012; Stretsolva et al., 2010; Urgen, Plank, Ishiquoro, Poizner, \& Saygin, 2013). Mu alpha and beta ERD was significantly stronger while watching actual swallowing compared to when watching the scrambled (kaleidoscope) condition.

Because mu ERD followed the same pattern in perception as it did in the production of swallowing, it seems logical to address the similarities between the two tasks. While the role of the involvement of the mirror system can be speculated, especially considering that beta ERD (i.e., motor activity) was clearly found in a sensory task, a possible explanation for the current findings is that watching swallowing may have elicited in participants the tendency to covertly replay of the sequence of swallowing movements as they watched them. Although, it was not possible to determine whether participants actively or passively engaged in covertly replaying the sequence of swallowing movements as they watched the swallowing video. Similar to other studies that have investigated that have investigated imagined movements (Neuper \& Pfurtscheller, 1999; Pfurthscheller et al., 2006) and watching biologically relevant movements have produced similar findings of activity from motor regions. This study provides further evidence that covert activity (while watching swallowing) can induce similar sensorimotor activity (e.g., internal modeling) to that seen during the performance of swallowing. It should be noted that (relative to baseline) the kaleidoscope condition produced low levels of mu ERD, however, considering the non-biological nature of the stimulus, it is doubtful that participants covert replayed the stimulus. It is more likely that this ERD resulted from the simple monitoring or attention to the stimulus which has been found in previous studies (Klimesch, Doppelmayr, Russegger, Pachinger, Schwaiger, 1998; Sauseng et al., 2005). However, attention was required during both the perceptual swallowing and the kaleidoscope conditions. Therefore, the statistical differences observed in the ERSP between observing the swallowing and the kaleidoscope are best attributed to the biological relevance and reproducible nature of the swallowing stimulus. 


\section{Mu-alpha and Beta: Swallowing Perception vs. Swallowing Performance}

Statistical (i.e., ERSP analyses) comparisons between perception and production conditions were not conducted because of a greater proportion of overall variance in the production conditions being accounted for by EMG activity. According to McMenamin (2011), this has the potential to reduce the spectral power in EEG neural components and potentially invalidate statistical comparisons between and production conditions.

Therefore, perceptions vs. production comparisons are descriptive in nature only. Overall, the perception of swallowing reflected similar spatial and temporal patterns activity when compared to the performance of swallowing. Although, the power of mu-alpha and beta ERD during the perception of swallowing was weaker than the power of mu-alpha and beta ERD during the performance of swallowing, especially during the most robust period of mu ERD during the later pharyngeal and esophageal phases. These results are consistent with Jenson et al. (2014) during a speech production motor imagery (i.e. covert syllable production task) in which weaker mu ERD was found as compared to mu ERD during the overt speech production tasks; although, the authors acknowledged that it was not possible to know whether all participants imagined themselves producing the syllables (2014). Additional studies involving the perception and performance of reaching and grasping movements have also reported similar results (Hari et al., 1998; Hari, 2014; Southgate, 2009).

Critical questions remain unanswered regarding the functional significance of $\mathrm{MN}$ activity during action observation. According to Iacoboni and colleagues, MN activity provides a flexible interface between feedforward and feedback driven 'internal model loops' by incorporating the perceived visual representation of the motor movements demonstrated by another into one's own predictive efference motor copy of movements (1999). In accordance with this view, it therefore seems reasonable to suggest that eliciting sensorimotor activity during the observation of swallowing may serve to reinforce one's efference copy of swallowing movements. In doing so, perhaps one's metacognitive awareness of swallowing could be used to reinforce therapeutically one's internal model representation of swallowing during efforts to acquire, refine, or restore swallowing. Thus, further investigation into the potential of an observation based therapy aimed at strengthening an individual's metacognitive awareness of sensorimotor processing during swallowing is warranted.

\section{General Discussion}

Overall, the results of this study showed that applying ICA to whole-head EEG data successfully identified mu components during the performance and perception of swallowing. Although slightly elevated levels of RV were found, these levels generally aligned with the levels of RV reported in a previous study that used the same techniques to measure SMI across speech perception and production tasks (Jenson et al., 2014). Across the perception and performance of swallowing, similar spatial and temporal cortical patterns of mu-alpha and beta ERD were observed. These findings are consistent with previous MEG and fMRI swallowing studies which have noted similar patterns of 
SMI during the observation of swallowing, orofacial movements such as biting and lip smacking, and visual speech. However, to our knowledge this is the first study to use EEG to map SMI in real time across the perception and performance of swallowing in addition to mapping muscle activity as a marker to help distinguish between oral and pharyngeal stages of swallowing. Considering the lack of precedence for interpreting patterns of mu-alpha and beta ERD obtained using EEG/ICA techniques during swallowing, and considering the shared attributes of speech and swallowing, it seemed important to compare the results of this study to the results of EEG studies involving speech perception and production.

The findings of simultaneous mu-alpha and beta ERD is during swallowing in the participants tested may be considered evidence of sensorimotor integration in the "normal" swallow. However, many swallowing disorders (i.e., dysphagia) include compromised SMI and considering that there is a paucity of measures to index SMI in real time during swallowing, the findings may have clinical implications. For example, patients with dysphagia due to a stroke involving the middle cerebral artery (MCA), which supplies the premotor and primary sensorimotor cortices may exhibit stronger patterns of mu ERD, because these regions are considered the main 'online cortical regulators' of swallowing movements (Ertekin \& Aydogdu, 2003). The PMC and primary motor and somatosensory cortices are thought to provide critical feedforward and feedback driven SMI, which allows for online adjustments to be performed when an unexpected event occurs during swallowing or a 'perturbations' in the sequence of swallowing movements occurs (Ertekin \& Aydogdu, 2003; Hamdy et al., 1999; Houde \& Nagarajan, 2011; Martin et al., 2001). For example, online adjustments in the strength and timing of swallowing movements are necessary to safely swallow an unexpectedly large bolus of food., Individuals with dysphagia due to neurological injuries affecting the PMC and primary somatosensory cortices, stronger patterns of mu ERD may be observed during swallowing in order to motorically compensate for sensory 'feedback prediction errors' (Houde \& Nagajaran, 2011). Questions such as these regarding how deviations from normal SMI would be reflected in patterns of mu ERD during swallowing remain unanswered. However, the results of this study suggest that using the novel techniques performed in this study offer exciting opportunities to measure functional changes in mu ERD during swallowing, which may be interpreted to understand the effects that various phase-specific (oral vs. pharyngeal and esophageal) swallowing impairments have upon the integrity of feedforwad and feedback driven SMI during swallowing.

In addition to mapping the functional dynamics of SMI exhibited by individuals with swallowing disorders, it could also be expected that peripherally stimulating sensory and motor activity via traditional swallowing exercises, feeding trials and thermal-tactile stimulation techniques would increase the timing and strength of mu ERD. In doing so, perhaps a better understanding may be obtained regarding the therapeutic effects of traditional sensorimotor swallowing treatments upon cortical SMI during swallowing. Furthermore, considering the evidence presented in this study along in conjunction with previous reports of similar patterns of cortical SMI during the perception and performance of swallowing, perhaps the development of an observation based therapy may be used to stimulate sensorimotor activity in the PMC and primary sensorimotor 
cortices by raising an individual's metacognitive awareness of sensorimotor processing during swallowing.

\section{Limitations and Future Directions of Study}

To our knowledge, this is the first study to use whole-head EEG to analyze dynamic patterns of SMI via mu rhythm ERS/ERD during swallowing. However, muscle artifacts are known to reduce the signal-to-noise ratio and tend to overwhelm the spectral characteristics of neural signals during the performance of tasks that require movement (McMennamin et al., 2011; Onton et al., 2012). As such, perhaps including additional electrodes in the future may help preserve the power of neural EEG signal data. In the current study, ECD localization techniques provided information regarding the average dipole source location of sensorimotor activity; however, performing additional analysis using sLORETA would provide a more sensitive means for mapping the spatial parameters of neural activity and would further validate average ECD estimates of neural activity (Bowers et al., 2013; Jenson et al., 2014). Another improvement might be seen by performing additional analyses of IC clusters exhibiting mu-alpha and beta activity in the frontal, temporal, and parietal lobes The inferior and poster regions of the parietal lobes have been shown to activate during swallowing, and are thought play an important role in interpreting reaffarent somatosensory feedback. Additionally, the inferior parietal lobules are considered part of the 'extended' MN network, and have been found to activate during the observation of hand and mouth movements (Chong, cunnington, Williams, Kansisher, \& Mattingley, 2008; Pindeda, 2008; Montgomery, Isenberg, \& Haxby, 2007). Since the parietal components were not the focus of the current study, they were not included, however, significant patterns of mu alpha and beta ERD localized to the parietal cortices were observed during the performance of swallowing, and extremely robust patterns of parietal mu ERD were found during the observation of swallowing. These findings warrant further investigation to better understand the role of the parietal lobes during swallowing, but are not surprising considering that sensory integration is known to occur in the parietal cortex. Additionally, clusters localized to the insula and anterior cingulate cortex are of interest. Considering that swallowing is known to activate a diverse network of cortical sensorimotor regions, perhaps including EEG coherence measures of functional connectivity to characterize the swallowing neural network would provide additional information regarding functional changes in the SMI during normal and dysfunctional swallowing (Milz, Faber, Pascual-Marqui, 2014).

Swallowing involves muscles of the face, neck, and vocal tract, and therefore future studies may benefit form included additional electrodes to better mark physiological events during the oral preparatory vs. oral transit vs. pharyngeal vs. esophageal phases of swallowing. For example, placing additional sEMG electrodes to the masseter muscles would allow for recording additional chewing movements during the oral preparatory phase, and would expand the view of SMI during the oral phase. Likewise, placing additional electrodes to capture submental activity would provide a more precise measure of SMI as swallowing transitions from the oral phase to the pharyngeal phase. Finally, self-administration of water boluses precluded including 
valuable data obtained during the oral preparatory phase due to the overwhelming muscle artifacts associated with hand and arm movements. Therefore, devising a hands-free method of bolus delivery in the future would also improve the likelihood of analyzing SMI during the oral preparatory phase.

During the oral transit and pharyngo-esophageal phases of swallowing, mu-alpha was found during the performance of swallowing; however, the techniques used in the current study did not allow for a determination as to whether mu-alpha ERD reflected sensory predictions of swallowing (i.e. feedforward), sensory feedback resulting from swallowing (i.e. feedback), or a combination of both. Similarly, the results did not allow for a determination of whether mu-beta ERD was associated with activating 'efference motor copies' of swallowing movements or simply reflected a continuous outflow of motor corrections. However, considering the findings of mu ERD localized to the PMC, which is proposed to mediate feedforward and feedback driven sensorimotor information, and the co-occurrence of alpha suppression with beta suppression within the same mu clusters, it is logical to interpret alpha suppression as an index of sensory feedback to the PMC (Jenson et al., 2014).

Additionally, In the future, including a "no-go" 'preparatory phase' where participants prepare to swallowing, but are instructed not to overtly swallowing when receiving a "no-go" cue may help to elucidate the timing and strength of mu ERD prior to the performance of swallowing. Similarly, expanding trials to allow for examination of the typically observed 'beta' rebound would also provide valuable information regarding the functional role of SMI after completing swallowing. Nonetheless, this preliminary study successfully mapped real time changes in mu-alpha and beta ERD across swallowing, thereby providing a functional map of SMI during the oral vs. pharyngeal phase of swallowing.

Another future direction of study may be to change the selected group of muscles that were monitored during swallowing using sEMG. The infrahyoid or laryngeal strap muscle group was chosen to temporally demarcate muscle activity during the pharyngeal phase of swallowing. This was done to in an attempt to completely dissociate oral phase SMI from pharyngeal phase SMI. As an alternative, selecting the suprahyoid muscle complex in the future may provide a slightly more precise measure of the 'onset' of pharyngeal phase activity (Vaiman et al., 2004). Finally, recent studies suggest differences in the strength and timing of swallowing physiology (Logemann et al., 2000; Robbins et al., 2008) and the degree of cortical sensorimotor activity during swallowing (Malandraki, Perlman, Karampinos, Sutton, 2010; Humbert \& Robbins, 2011) in individuals over the age of 65 . Considering that dysphagia typically affects older individuals, an important next step would be to use this technique to analyze the functional dynamics of cortical SMI exhibited by an older cohort of normal adults. 


\section{Conclusions}

Overall, addressing the aims of this study lead to several conclusions regarding the use of EEG to measure SMI during swallowing, the functional role of SMI during swallowing, and relationship between the perception and performance of swallowing. First, applying ICA to whole head EEG data during the performance and perception of swallowing revealed mu clusters of IC's exhibiting significant mu ERD during the perception and performance of swallowing. Second, time-frequency analyses of mualpha and mu-beta ERD revealed functional measures of SMI in real time across swallowing. Third, concurrently measuring EMG activity in the infrahyoid cluster provided a reliable physiological (i.e. temporal) marker to delineate SMI during the oral vs. the pharyngeal and esophageal phases of swallowing. Fourth, evidence of significantly stronger patterns of mu alpha and beta ERD during the performance of swallowing when compared to tongue-tapping provided evidence supporting the notion that mu-alpha and beta ERD measure higher order SMI, as opposed to providing isolated measures of cortical sensory and motor activity localized to the PMC. Finally, evidence of similar patterns of SMI during the perception and performance of swallowing supports hypotheses regarding a possibility of a functional connection between the swallowing perception and swallowing performance, and supports further investigation into the therapeutic potential for developing an observation-based swallowing therapy. 


\section{LIST OF REFERENCES}

Alomar, M.H., Samaha, A., and Alkamha, K. (2013). Automated classification of L/R hand movement EEG signals using advanced feature extraction and machine learning. International Journal of Advanced Computer Science and Applications, 4

Arnstein, D., Cui, F., Keysers, C., Maurits, N.M., and Gazzola, V. (2011). Mu suppression during action observation and execution correlates with BOLD in dorsal premotor, inferior parietal and SI cortices. Trends in Neuroscience, 31, $14243-14249$

Ayers, A.J. (1972). Sensory Integration and the child. Los Angeles: Western Psychological Services.

Ayers, A.J. (1989). Sensory Integration Praxis Tests Manual. Los Angeles: Western Psychological Services

Bai, O., Mari, Z., Vorback, S., and Hallett, M. (2005). Asymmetric spatiotemporal patterns of event-related desynchronization preceding voluntary sequential finger movements: a high-resolatuion EEG study. Conical Neurophysiology $116,1213-1221$.

Bebo, N., Ribary, U., and Ward, L. (2014). Fast dynamics of cortical functional and effective connectivity during word reading. PLOSone

Bowers, A., Saltuklaroglu, T., Harkrider, A., and Cuellar, M. (2013). Suppression of the mu rhythm during speech and non-speech discrimination revealed by independent component analysis: implications of sensorimotor integration in speech processing. PLOS One 8, e72024.

Chen, Bin, Daly, Gao (2013). Event related desynchronization (ERD) in alpha band during a hand mental rotation task. Neuroscience Letters, 541, 28-42

Cheyne et al. (2003) Neuromagnetic imaging of cortical oscillations accompanying tactile stimulation. Cognitive Brain Research, 17, 599-611

Chuang, C., Ko, L., Jung, T., Lin, C. (2014) Automatic design for independent component analysis based brain-computer interfacing. Journal of Neural Engineering, 10.

Crone, Boatman, Gordon, and Hao (2001). Induced electrocorticographic gamma activity during auditory perception. Clinical Neurophysiology, 112, 565-82

Delorme, A., and Makeig, S. (2004). EEGLAB: an open source toolbox for analysis of single- trial EEG dynamics including independent component analysis. Journal of Neuroscience Methods, 134, 9-21. 
Delorme, A., Sejnowski, T., and Makeig, S. (2007). Enhanced detection of artifacts in EEG data using higher-order statistics and independent component analysis. Neuroimage, 34, 1443-49

Ding, R., Larson, Logemann, and Rademaker. (2001). Surface electromyographic and electroglottographic studies in normal studies under two swallow conditions: normal and during the Mendelsohn maneuver. Dysphagia, Vol. 17

Ertekin and Aydogdu (2003). Neurophysiology of swallowing. Clinical Neurophysiology $114, \quad 222-2244$.

Furlong, R., Hobson, A., Aziz, Q., Barnes, G., Singh, K., Hillbrand, A., Thompson, D., and Hamdy, S. (2004). Dissociating the spatio-temporal characteristics in the healthy adult brain of cortical neuronal activity associated with human volitional swallowing in the healthy adult brain. Neuroimage, 22, 1447-1455.

Gaetz, W., and Cheyne, D. (2006). Localizaiton of sensorimotor cortical rhythms induced by tactile stimulation using spatially filtered MEG. Neruoimage, 30, 899-908

Gaetz, W., Macdonald, M., Cheyne, D., and Snead, O.C. (2010). Neuromagnetic imaging of movement-related cortical oscillations in children and adults: age predicts post-movement beta rebound. Neuroimage, 51, 792-807.

Gwin, J.T., Gramann, K., Makeig, S. (2010). Removal of movement artifact rom high density EEG recording durign walking and running. Journal of Neurophysiology

Hamdy, Rothwell, Hobson, Barlow, and Thompson (1999). Identification of the cerebral loci processing human swallowing with H2o15 PET activation. Journal of Neurophysiology81, 1917-1926.

Hari, R (2006). Action-perception connection and the cortical mu rhythm. Progress in Brain Research 159, 253-260.

Humbert, I.A., and Robbins, J.A. (2007). Normal swallowing and functional magnetic resonance imaging: a systematic review. Dysphagia, 22, 266-275

Humbert, I.A., Lockarnde, A., Christopherson, H., German, R., Stone, A. (2012). Adaptation of swallowing hyo-laryngeal kinematics is distinct in oral versus pharyngeal sensory processing. Journal of Applied Physiology

Humbert, I.A. and German, R.Z. (2013). New directions for understanding neural control in swallowing: The potential and promise of motor learning. Dysphagia

Jean A. (1984). Braintem organization of the swallowing network. Brain Behavior 25, 109-116. 
Jean A. (2001). Brain stem control of swallowing: neuronal network and cellular mechanisms. Physiological Reviews 81, 929-969

Jensen, Goel, Kopell, Pohja, Hari, and Ermentrout. (2005). On the human sensorimotor cortex beta rhythm: sources and modeling. NeuroImage, 26, 347-5

Jensen, O., Kaiser, J., and Lachaux, J.P. (2007). Human gamma-frequency oscillations associated with attention and memory. Trends in Neurosciences 30, 317-324.

Jenson, D., Bowers, A., Harkrider, A.W., Thornton, D., Cuellar, M., and Saltuklaroglu, T., (2014). Temporal dyanmnics of sensorimoro integration in speech perception and production: independent component analysis of EEG data. Frontiers in Psychology, 5, 656

Kalaska, Scott, Cisek, \& Sergio (1977). Cortical control of reaching movements, Current Opinion in Neurobiology, 7, 849-59

Kamisaruk, B.R., Mosier, K.M., WenChing, L., Criminale, C., Zabrorskzky, L., Whipple, B., and Kalnin, A. (2002). Functional localization of brainstem and cervical spinal cord nuclei in humans with fMRI. Americal Journal of Neuroradiology 23, 609617.

Kawato, M. (1999). Internal models for motor control and trajectory planning. Current Opinion in Neurobiology, 9, 718-27

Kilivik, B.E., Azepffel, M., Brovelli, A., Mackay, W.A.,and Riehle, A., (2013). The ups and downs of beta oscillation sin the sensorimotor cortex. Experimental Neurology 245, 15-25.

Lau, T.M., Gwin, J.T., and Ferris, D.P. (2014). Walking reduces sensorimotor network connectivity compared to standing. Journal of Neuroengineering and rehabilitation 11,14 .

Langmore, S.E. Endoscopic evaluation and treatment of swallowing disorders. United States: Theime medical Publishers, Inc. 2001. Print

Lenartowicz, A, Escobedo-Quiroz, R., and Cohen, J., D., (2010). Updating of context in working memory: an event related potential study. Cognition, Affect, Behavioral Neuroscience, 13

Logemann, J.A. Evaluation and treatment of swallowing disorders, Second Edition. Unites States: Pro-Ed, 1998. Print 
Lv, J., Yuanquing, L, Zhengui, G. (2010). Decoding hand movement velocity from electroencephalograph signals during a drawing task. Biomedical Engineering, 9, $1-21$

Makeig, S., Debener, S., Onton, J., and Delorme, A. (2004). Mining event related brain dynamics. Trends in Cognitive Science 8, 204-210.

Malandraki, G.A., Perlman, A.L., Karampinos, D.C., Sutton, B.P. (2011). Reduced somatosensory activations in swallowing with age. Human Brian Mapping, 32, $730-43$

Martin, R.E., Goodyear, B.G., Gati, J.S., Menon, R.S. (2001). Cerebral cortical representation of automatic and volitional swallowing in humans. Journal of Neurophysiology, 85, 93-950

Martin, R.E., Macintosh, B.J., Smith, R.C., Barr, A.M., Stevens, T.K., Gati, J.S., Menon, R.S. ( 2004). Cerebral areas processing swallowing and tongue movement are overlapping but distinct: a functional magnetic resonance imaging study. Journal of Neurophysiology 92, 2428-2493

Malendraki, Sutton, Perlman, and Karampinos (2010). Age related differences in laterality of cortical activations in swallowing. Dysphagia, 25, 238-249.

McFarland, Sarnacki, Vaughan, Wolpaw (2000). Mu and beta rhythm topographies during motor imagery and actual movement. Brain Topography, 3, 177-186.

McMenamin, B.W., Shackman, A.J., Greischar, L.L., and Davidson, R.J. (2011). Electromyogenic artifacts and electroencephalographic Inferences Revisited. Neuroimage 54, 4-9.

Mosier, R.E., Patel, R., Liu, W.C., Kalnin, A., Maldijian, J, Baredes, S. (1999). Cortical representation of swallowing in normal adults: functional implications. Laryngoscope, 109, 1417-1423

Mosier, K., Bereznaya, L., (2001) Parallel cortical networks for volitional control of s wallowing in humans, Experimental Brain Research. 40(3) 280-289.

NINDS Swallowing Disorders Information Page. (2014, February 1). Retrieved March 1, 2014.

Neuper, C., Wortz, M., and Pfurtshceller, G. (2006). ERD/ERS patterns reflecting sensorimotor activation and deactivation. Progress in Brain Research 159, 211222.

Obai, Mari, Vorbach, and Hallett, (2005). Complex strucures and common dynamics of self-awareness. The Dynamics of Agency and Ownership in Motor Action. 
Onton, J., Westerfield, M., Townsend, J., Makeig, S. (2006). Imaging human EEG dynamics using independent component analysis. Neuroscience Biobehavioral Reviews 30, 808-822.

Paek, A.Y., Harshavardhan, A., Conreras-Vidal, J. (2014). Decoding repretitive finger movements with brain activity acquired via non-invasive electroencephalography. Frontiers in Neuroengineering

Palva and Palva. (2010). Functional roles of alpha-band phase synchronication in local and large scale cortical networks. Frontiers in Psychology, 4, 186

Perlman, Palmer, McCulloch, and VanDaele. (1999). Electromyographic activity from human laryngeal, pharyngeal, and submental muscles during swallowing. Journal of Applied Physiology, Vol. 86

Pearlman, Schulze-Delrieu. Deglutition and its disorders. United States: Singular Publishing Group. 1997. Print

Pfurtscheller, G, and Lopes Da Silva, F.H. (1999). Event-related EEG/MEG synchronization and desynchronization: basic principles. Clinical Neurophysiology 110, 1842-1857

Pfurtscheller, G, Stancak, A., Jr., and Neuper, C. (1996). Post-movement beta synchronization. A correlate of an idling motor area? Electroencephalography Clinical Neurophysiology 98, 281-293.

Pineda, J.A. (2005). The functiona significance of mu rhythms: translating "seeing" and "hearing" into "doing." Brain Research Reviews 50, 57-68.

Pineda, J.A. (2008). Sensorimotor cortex as a critical component of an 'extended' mirror neuron system: does it solve the development, correspondence and control problems in mirroring? Behavioral Brain Function, 4.

Quandt, L., Marshall, P., Goldin-Meadow, S., (2012). Sensitivity of alpha and beta oscillations to sensorimotor characteristics of action: An EEG study of action production and gesture observation. Neuropsychologia, 50, 2745-51

Rao, S.M., Binder, J.R., Bandettinin, P.A., Hammeke, T.A., Yetkin, F.Z, Jesmanowicz, A (1993). Functional magnetic resonance imaging of complex human movements. Journal of Neurological Science, 140, 67-74

Shaw, S.M., and Martino, R. (2013). The normal swallow: muscular and neurophysiological control. Otolaryngology Clinics of North America, 46, 937965 
Shelenz P.D., Klasen, M., and Mathiak, K. (2013). Multisensroy integration of dynamic emotional faces and voices: method for simultaneous EEG-fMRI measurements. Frontiers in Human Neuroscience, 7, 729.

Siegel, M., Donner, T.H., and Engle, A.K. (2012). Spectral fingerprints of large scale neuronal interactions. Nature Neuroscience Reviews, 13, 124-134

Tzagarakis, C., Ince, N., Leuthold, A.C., and Pellizzer, G. (2010). Beta band activity during motor planning reflects response uncertainty. The Journal of Neuroscience, 30, 11270-11277

Vaiman, Eviatar, and Segal, (2004). Evaluation of normal deglutition with the help of rectified surface electromyography records. Dysphagia, Vol. 19

Vaiman, Segal, and Eviatar, (2005). Surface eletromyography of continuous drinking in healthy adults. Laryngoscope, Vol. 115

Vaiman (2009). Standardization of surface electromyography utilized to evaluate patients with dysphagia. Head and Face Medicine, 3

Wagner, Escalante, Grieshofer, Neuper, Muller-Putz, and Scherer. (2012). Level of participation in robotic-assisted treadmill walking modulates midline sensorimotor EEG rhythms in able bodied subjects. Neuroimage, 15, 1203-11

Wolpert, D.M., Diedrichsen, J., and Flanagan, R. (2011). Principles of sensorimotor learning. Nature Neuroscience Reviews, 12, 739-51

Zaepffel, M., Trachel, R., Kilavik, B.E., and Brochier, T. (2013). Modulations of EEG beta power during planning and execution of grasping movements. PLOS One 8.

Zald and Pardo (2001). The functional neuroanatomy o voluntary swallowing. Annals of Neurology, 46, 281-286 


\section{VITA}

Megan Cuellar was born in 1979 in Memphis, Tennessee. She graduated from Briarcrest High school and received her Bachelor's Degree from the University of Tennessee in 2003. She went on to earn her Master's Degree from the University of Memphis in 2005. Megan will receive her Ph.D. from the University of Tennessee Health Science Center Speech and hearing Science Program in December 2014. Megan Cuellar is an assistant professor at Midwestern University in in the Department of Speech Pathology, and specializes in the neuroimaging and the neurophysiology of swallowing and motor speech disorders. She currently conducts research using EEG neuroimaging techniques to better understand the way in which the brain controls swallowing, speech, language, and other neurocognitive processes. 